\title{
PRICING PRECIPITATION BASED DERIVATIVES
}

\author{
RENÉ CARMONA AND PAVEL DIKO
}

\begin{abstract}
We consider the problem of pricing a derivative contract written on the precipitation at a specific location during a given period of time. We propose a jump Markov process model for the stochastic dynamics of the underlying precipitation. Our model is based on pulse Poisson process models widely used in hydrology. We develop maximum likelihood parameter estimation procedures to fit our model to rainfall data. In order to price derivatives, we assume the existence of a traded asset whose price dynamics are influenced by the precipitation at the location in question, and we rely on the utility indifference approach. Two utility functions are considered: exponential and power utility. We derive explicit solutions for the exponential and bounds for the power utility.

Finally, we apply our model fitting and pricing techniques to a sample rainfall contract in Norway.
\end{abstract}

\section{INTRODUCTION}

In this paper we concentrate on a specific class of derivatives written on non-traded assets, precipitation derivatives. They are part of a larger group of weather derivatives used for weather risk mitigation in a wide array of businesses ranging from agriculture to the entertainment industry.

Pricing of precipitation derivatives poses a great challenge compared to the other types of weather derivatives, let alone classical equity derivatives. From a practical view point, precipitation can be quite a localized phenomenon. Theoretical difficulties lie in the fact that, despite the existence of intensive hydrological research has been conducted in this area for more than 40 years, it is not straightforward to develop a tractable mathematical model accurately representing the reality of the precipitation at a specific location.

Moreover, once a model for the precipitation has been established, a second source of difficulty arises: it is necessary to develop a pricing methodology which can incorporate the idiosyncracies of the underlying precipitation process. Since precipitation cannot be traded directly, the market model is most likely to be incomplete. Instead of searching for an appropriate risk neutral probability measure, we follow the utility indifference pricing approach to derive buyer and seller's prices. This valuation method can be described in a few sentences as follows. Let us consider an economic agent whose time-invariant risk preferences are given by a utility function. Given an initial wealth, she purchases (resp. sells) a derivative written on a non-traded asset, and she uses her endowment after the transaction to set up a portfolio in a bank account and shares of a traded asset and follow a selffinancing strategy, re-balancing her portfolio in continuous time. She searches for an optimal strategy which maximizes her expected utility of wealth at maturity of the derivative. The expected utility of terminal wealth can also be computed had she not entered the transaction. The buyer's (resp. seller's) price of the derivative is set in such a way as to make the agent indifferent between the two scenarios - hence the name utility indifference pricing given to this valuation method.

Date: February 28, 2005. 
The topic of utility indifference pricing was first introduced by Hodges and Neuberger in the early nineties, but it had to wait almost an entire decade to catch on. It was rediscovered by Davis [?] and used by Henderson [?, ?], and Musiela and Zariphopoulou [?, ?, ?] who considered the case of a derivative written on a non-traded asset whose price dynamics are given by a geometric Brownian motion. Most of these contributions are restricted to the case of the exponential utility function, for in the case of the power utility, one is only able to obtain bounds on the prices. More generally, Zariphopoulou considers in ([?]) the problem of utility optimization when the influence of the non-traded asset on the traded asset comes through both the correlation between the driving Wiener processes and the coefficients of the price process SDE. She uses power utility and obtains results for the scenario without the derivative present. Finally, we mention that Musiela and Zariphopoulou gave an abstract form of the indifference pricing paradigm on a tree, and that Carmona and Danilova ([?]) derived the indifference price for a claim on a non-traded quantity (temperature) whose dynamics is driven by a diffusion process when allowed to invest in traded assets whose dynamics are given by diffusions whose coefficients depend upon the non-traded asset, and when the agent has exponential utility function. The interested reader is referred to the forthcoming book [?] for a detailed account of the state of the art in indifference pricing.

The paper is naturally divided into three parts. In the first part, we develop a model for the precipitation process, and we find maximum likelihood estimators for its parameters. In the second part, we adapt utility indifference pricing to a class of security price models including the precipitation model from part one. We consider exponential and power utility functions. For the exponential utility we obtain an explicit formula for the price of the derivative, while for the power utility, we can only prove lower and an upper bounds. Finally, we conclude the paper with the detailed analysis of a practical example. Except for the highly publicized example of the three year deal offered by an amusement park in Paris, not much public information is available on the terms and conditions of precipitation options and their prices. The example we propose in the last section of the paper is made up. However, we used our personal experience to set it up to make it as realistic as possible.

\section{Modeling Rainfall Dynamics}

2.1. Precipitation Data. Data serving as underlying for precipitation contracts come from meteorological stations around the globe. Although made in discrete time intervals, the observations come close enough to complete continuous time observation of the rainfall intensity. A period of rainfall, when its intensity stays constant, can be described by a pair $(\xi, \beta)$ of real numbers. Here $\beta>0$ is the length of time for which the rainfall intensity stays constant and equal to $\xi>0$. Let us denote $M$ the sequence $\left\{\left(\xi_{i}, \beta_{i+1}\right)\right\}_{i=1,2,3, \ldots, n}$ of pairs describing the consecutive periods of constant rainfall intensity. The sequence $M$ comprises the statistical data for which we want to formulate a parsimonious yet well-fitting probabilistic model.

Precipitation has been the subject of intensive research for years. Several types of models have been developed [?], which can be divided into four categories: meteorological models which seek to capture the dynamics of the large scale atmospheric processes controlling precipitation [?], multiscale models which use multifractal cascades to describe rainfall [?, ?], statistical models which use purely statistical techniques to fit the rainfall data to well known distribution types with little emphasis on underlying physical processes [?] and last stochastic processes based models which try to describe 
the rainfall behavior by a small set of physically meaningful parameters driving a stochastic process. They were introduced by LeCam in 1961 [?] and further developed by Rodriguez-Iturbe et al. [?, ?].

2.2. Precipitation Model. A version of the Bartlett-Lewis Poisson-cluster (BLPC) process model of Rodriguez-Iturbe, Cox and Isham [?] forms the basis of the model which we propose. In this model, rainfall is assumed to be composed of storms which are in turn composed of rainfall cells. The storms arrive according to a Poisson process; within each storm, cells arrive according to another Poisson process and the duration of the activity of the storm is random. Each cell has random duration and random depth. Both storms and rain-cells can overlap. If two or more cells overlap their depths add up. The best data-fitting version of the model uses exponential distributions for both the storm and cell duration ([?, ?]). Although the BLPC model is physically intuitive, methods for fitting its parameters are quite limited and predominantly not very stable. The most widely used approach is based on 'method-of-moments' type fitting as, for example, in Rodriguez-Iturbe et al. ([?]). In what follows, we reformulate the Rodriguez-Iturbe model, and we explain why the new model keeps all the important features of the old one, while allowing for maximum likelihood estimation (MLE for short) of parameters. Only then, do we state a rigorous definition of the new model.

Main obstacles to application of MLE to BLPCs model are the "physically appealing but mathematically intractable dependencies involved between the rainfall intensities at different time points," [?]. Although intractable, the model is very close to being Markovian. There are two properties which compromise the Markov property: first, the rectangular shape of the cells implies the memory of the depths of all active cells at any time and second, the rate of cell extinction at a given time depends on the number of active cells which is unobservable. The first issue can be dealt with in the following way: at a cell arrival time, the rainfall process jumps up by a random amount and at the cell's extinction time, it jumps down by a random amount with appropriate distribution. If on average the jumps up are as large as the jumps down, one can call such a modified model a 'randomization' of the rectangular cell shape. There is one technical point to be addressed - the possibility of a jump to the negative region. The second issue is also resolvable. The rate of extinction can be made dependent on the instantaneous intensity of the rainfall and not the number of active cells. Again this can be thought of as "smoothing" of the Rodriguez-Iturbe BLPC model. It is noteworthy to realize that in the case of deterministic cell depths, this approach is identical to the BLPC model mechanics.

We propose to model rainfall intensity within a single storm by a homogeneous jump Markov process $\left\{Y_{t}\right\}$ whose infinitesimal generator $G$ is given by:

$$
[G \phi](x)=\int_{\mathbb{R}_{+}}(\phi(y)-\phi(x)) A(x, y) \nu(d y),
$$

where:

$$
\begin{aligned}
A(x, y)=\left\{\lambda_{1} \lambda_{u} e^{-\lambda_{u}(y-x)} 1_{(x, \infty)}(y)\right. & \left.\bar{\lambda}_{2}(x) \lambda_{d} e^{-\lambda_{d}(x-y)} 1_{(0, x)}(y)+\bar{\lambda}_{2}(x) e^{-\lambda_{d} x} 1_{\{0\}}(y)\right\}
\end{aligned}
$$

with

$$
\bar{\lambda}_{2}(\xi)=1_{(0, \infty)}(\xi)\left(\lambda_{2}^{(I)}+\lambda_{2}^{(I I)} \kappa(\xi)\right)
$$


where $\nu$ is Lebesgue's measure on $[0, \infty)$ with an added unit point mass at 0 , and $\kappa(\xi)$ is defined on $\mathbb{R}_{+}$and satisfies $\kappa(x)=x$ for $0 \leq x \leq K$ (for some large $K$ ), it is bounded, it is 3 -times differentiable, $(\partial / \partial x) \kappa(x)>0$. The model is determined by the parameter vector $\left(\lambda_{1}, \lambda_{2}^{(I)}, \lambda_{2}^{(I I)}, \lambda_{u}, \lambda_{d}\right)=$ $\left(\theta_{1}, \theta_{2}, \theta_{3}, \theta_{4}, \theta_{5}\right)=\theta \in \Theta=\mathbb{R}_{+}^{5}$.

2.3. Maximum Likelihood Parameters Estimates. The advantage of having a Markovian model for the single storm is the convenience of being able to write the likelihood function. As stated in Ubsection 2.1, we observe $M=\left\{\left(\xi_{i}, \beta_{i+1}\right)\right\}_{i=1, \ldots, n}$. For the continuous time process $Y, \beta_{i+1}>0$ is the sojourn time in state $\xi_{i}>0 . M$ is an observation of the so called embedded Markov chain corresponding to the continuous time process $Y$ (see [?]). The log-likelihood function (with respect to the measure $\nu$ ) of a particular $M$ is then:

$$
L(M, \theta)=\ln \left[\prod_{i=1}^{n-1} F\left(\xi_{i}, \beta_{i+1}, \xi_{i+1}, \theta\right)\right],
$$

where:

$$
F(\xi, \beta, \eta, \theta)=f(\xi, \eta, \theta) q(\xi, \theta) e^{-q(\xi, \theta) \beta} .
$$

Intuitively, the value of $F$ is the product of two probabilities: $q(\xi, \theta) e^{-q(\xi, \theta) \beta}$ which is the density of the sojourn time $\beta$ of the jump Markov process $Y$ in the current state $\xi$, and $f$ which is the density of the distribution for the new state $\eta$ resulting from the next jump. For the storm model defined by (1):

$$
\begin{aligned}
f(\xi, \eta, \theta)=1_{[\xi, \infty)}(\eta) & \left(\frac{\lambda_{1}}{\lambda_{1}+\bar{\lambda}_{2}(\xi)} \lambda_{u} e^{-\lambda_{u}(\eta-\xi)}\right) \\
& +1_{(0, \xi)}(\eta)\left(\frac{\bar{\lambda}_{2}(\xi)}{\lambda_{1}+\bar{\lambda}_{2}(\xi)} \lambda_{d} e^{-\lambda_{d}(\xi-\eta)}\right)+1_{\{0\}}(\eta)\left(\frac{\bar{\lambda}_{2}(\xi)}{\lambda_{1}+\bar{\lambda}_{2}(\xi)} e^{-\lambda_{d} \xi}\right)
\end{aligned}
$$

and:

$$
q(\xi, \theta)=\lambda_{1}+\bar{\lambda}_{2}(\xi)
$$

We derive the values of the estimators by solving the system of first order conditions, and we check that the obtained solutions are indeed the unique maximum likelihood estimators of the model parameters. Denote $U=\left\{i ; \xi_{i+1}-\xi_{i}>0\right\}, D=\left\{i: \xi_{i+1}-\xi_{i}<0\right\}, D_{0}=\left\{i: \xi_{i+1}=0\right\}$, $\Xi_{+}=\left\{i: \xi_{i}>0\right\}$. The candidate estimators are then:

$$
\lambda_{d}=\frac{\left|D \backslash D_{0}\right|}{\sum_{i \in D}\left(\xi_{i}-\xi_{i+1}\right)}, \lambda_{1}=\frac{|U|}{\sum_{i=2}^{n} \beta_{i}}, \lambda_{u}=\frac{|U|}{\sum_{i \in U}\left(\xi_{i+1}-\xi_{i}\right)}
$$

and the solution of the system of equations:

$$
\begin{aligned}
\sum_{i \in D} \frac{1}{\lambda_{2}^{(I)}+\lambda_{2}^{(I I)} \kappa\left(\xi_{i}\right)} & =\sum_{i=2}^{n} \beta_{i} \\
\sum_{i \in D} \frac{\kappa\left(\xi_{i}\right)}{\lambda_{2}^{(I)}+\lambda_{2}^{(I I)} \kappa\left(\xi_{i}\right)} & =\sum_{i=1}^{n-1} \kappa\left(\xi_{i}\right) \beta_{i+1}
\end{aligned}
$$

The following proposition states the uniqueness of the estimates for $\lambda_{2}^{(I)}$ and $\lambda_{2}^{(I I)}$. 
Proposition 2.1. For any realization of $M$, if the set $D$ is non-empty and the system of equations (4) and (5) has a solution, then it is unique.

Proof. If we use the notation $B=\sum_{i=2}^{n} \beta_{i}$, and $C=\sum_{i=1}^{n-1} \kappa\left(\xi_{i}\right) \beta_{i}$, we get:

$$
\begin{aligned}
\Phi(a, b) & =\sum_{i \in D} \frac{1}{a+b \kappa\left(\xi_{i}\right)}-B \quad a, b>0 \\
\Psi(a, b) & =\sum_{i \in D} \frac{\kappa\left(\xi_{i}\right)}{a+b \kappa\left(\xi_{i}\right)}-C \quad a, b>0 .
\end{aligned}
$$

Since $D$ is non-empty, and since for every $i$ in $D \xi_{i}>0$, the function $\Phi$ is strictly decreasing both in $a$ and $b$. Therefore the equation $\Phi(a, b)=0$ defines an implicit function $a=a(b)$ for $b \in\left(0, \frac{1}{B} \sum_{i \in D} \kappa\left(\xi_{i}\right)^{-1}\right)$ which is smooth and:

$$
a^{\prime}(b)=-\frac{\partial \Phi / \partial b}{\partial \Phi / \partial a}=-\frac{\sum_{i \in D} \frac{\kappa\left(\xi_{i}\right)}{\left(a(b)+b \kappa\left(\xi_{i}\right)\right)^{2}}}{\sum_{i \in D} \frac{1}{\left(a(b)+b \kappa\left(\xi_{i}\right)\right)^{2}}} .
$$

Now, let us define $g(b)=\Psi(a(b), b) . g$ is only well-defined for $b \in\left(0, B^{-1} \sum_{i \in D} \kappa\left(\xi_{i}\right)^{-1}\right)$. The statement of the proposition is equivalent to saying that if $g(b)=0$ has a solution, then it is unique. It is easy to see that $g$ is smooth. To show the uniqueness we prove that $g^{\prime}<0$, and thus $g$ is strictly decreasing:

$$
\begin{aligned}
g^{\prime}(b) & =\sum_{i \in D} \frac{-\kappa\left(\xi_{i}\right)}{\left(a(b)+b \kappa\left(\xi_{i}\right)\right)^{2}}\left(a^{\prime}(b)+\kappa\left(\xi_{i}\right)\right) \\
& =\left(\sum_{i \in D} \frac{-\kappa\left(\xi_{i}\right)}{\left(a(b)+b \kappa\left(\xi_{i}\right)\right)^{2}}\right) \frac{\sum_{i \in D} \frac{-\kappa\left(\xi_{i}\right)}{\left(a(b)+b \kappa\left(\xi_{i}\right)\right)^{2}}}{\sum_{i \in D} \frac{1}{\left(a(b)+b \kappa\left(\xi_{i}\right)\right)^{2}}}-\sum_{i \in D} \frac{\kappa\left(\xi_{i}\right)^{2}}{\left(a(b)+b \kappa\left(\xi_{i}\right)\right)^{2}} \\
& =\frac{\left(\sum_{i \in D} \frac{\kappa\left(\xi_{i}\right)}{\left(a(b)+b \kappa\left(\xi_{i}\right)\right)^{2}}\right)^{2}-\left(\sum_{i \in D} \frac{\kappa\left(\xi_{i}\right)^{2}}{\left(a(b)+b \kappa\left(\xi_{i}\right)\right)^{2}}\right)\left(\sum_{i \in D} \frac{1}{\left(a(b)+b \kappa\left(\xi_{i}\right)\right)^{2}}\right)}{\sum_{i \in D} \frac{1}{\left(a(b)+b \kappa\left(\xi_{i}\right)\right)^{2}}} .
\end{aligned}
$$

The sign of the derivative only depends on the numerator since the denominator is positive. So we drop the denominator:

$$
\begin{aligned}
& \left(\sum_{i \in D} \frac{\kappa\left(\xi_{i}\right)}{\left(a(b)+b \kappa\left(\xi_{j}\right)\right)^{2}}\right)^{2}-\left(\sum_{i \in D} \frac{\kappa\left(\xi_{i}\right)^{2}}{\left(a(b)+b \kappa\left(\xi_{j}\right)\right)^{2}}\right)\left(\sum_{i \in D} \frac{1}{\left(a(b)+b \kappa\left(\xi_{j}\right)\right)^{2}}\right) \\
& \quad=\sum_{i j} \frac{\kappa\left(\xi_{i}\right) \kappa\left(\xi_{j}\right)-\kappa\left(\xi_{i}\right)^{2}}{\left(a(b)+b \kappa\left(\xi_{i}\right)\right)^{2}\left(a(b)+b \kappa\left(\xi_{j}\right)\right)^{2}}=\sum_{i<j} \frac{2 \kappa\left(\xi_{i}\right) \kappa\left(\xi_{j}\right)-\kappa\left(\xi_{i}\right)^{2}-\kappa\left(\xi_{j}\right)^{2}}{\left(a(b)+b \kappa\left(\xi_{i}\right)\right)^{2}\left(a(b)+b \kappa\left(\xi_{j}\right)\right)^{2}} \\
& \quad=-\sum_{i<j} \frac{\left(\kappa\left(\xi_{i}\right)-\kappa\left(\xi_{j}\right)\right)^{2}}{\left(a(b)+b \kappa\left(\xi_{i}\right)\right)^{2}\left(a(b)+b \kappa\left(\xi_{j}\right)\right)^{2}} \\
& \quad<0 . \quad \square
\end{aligned}
$$

To establish that the above estimators are consistent maximum likelihood estimators of the true model parameters, we need to verify certain smoothness properties of the transition density of the 
imbedded Markov chain for the process $Y$ in order to use the following theorem ([?]) of Billingsley which we state for the sake of completeness.

Theorem 2.2 (Billingsley). Suppose that the Markov process $\left\{Y_{t}\right\}$ defined by its infinitesimal generator (1), $f, q$ defined by (3) and the parameter space $\Theta$ satisfy the following conditions:

(1) $P\left(Y_{t+s}=x \mid Y_{t}=x\right)$ is a continuous function of $s$ for every $x$ and $t$, the sample paths of $Y_{t}$ are right-continuous and $q(\xi, \theta)$ is a bounded function for any $\theta \in \Theta$.

(2) For any $\xi$, the set of $\eta$ for which $f(\xi, \eta, \theta)>0$ does not depend on $\theta$.

(3) For any $\xi$, $\beta$ and $\eta$ the $F(\xi, \beta, \eta, \theta)$ (and thus $G(\xi, \beta, \eta, \theta)=\ln F(\xi, \eta, \theta)$ ) has continuous third order partial derivatives throughout $\Theta$.

(4) For any $\theta \in \Theta$ there is a neighborhood $N$ of $\theta$ such that for any three parameters $u, v, w$ and any state $\xi$ :

where $F_{u}, F_{u v}, F_{u v w}$ denote the first, second and third order partial derivatives with respect to parameters $\theta_{u}, \theta_{v}, \theta_{w}$ and $z_{1}$ is a random variable with the stationary distribution $\pi_{\theta}$ of the Markov chain $\left\{\xi_{1}, \xi_{2}, \xi_{3}, \ldots\right\}$ and the conditional distribution of $\left(\rho_{2}, z_{2}\right)$ given $z_{1}$ is determined by its density $F\left(z_{1}, \rho_{2}, z_{2}, \theta\right)$.

(5) There exists $\delta>0$ such that for all $u, E_{\theta}\left[\left|G_{u}\left(z_{1}, \rho_{2}, z_{2}, \theta\right)\right|^{(2+\delta)}\right]<\infty$ and $\sigma_{u v}(\theta)$ defined by $\sigma_{u v}(\theta)=E_{\theta}\left[G_{u}\left(z_{1}, \rho_{2}, z_{2}, \theta\right) G_{v}\left(z_{1}, \rho_{2}, z_{2}, \theta\right)\right]$ form a non-singular matrix $\sigma(\theta)=$ $\left(\sigma_{u v}(\theta)\right)$.

(6) For each $\theta \in \Theta$, there is exactly one stationary distribution $\pi_{\theta}(\cdot)$ for the imbedded Markov process $\left\{\xi_{n}\right\}$, and $\pi_{\theta}(\xi, \cdot) \ll \pi_{\theta}(\cdot)$ for each $\xi \in R_{+}$.

and that $\theta^{0} \in \Theta$ is the true value of the parameter vector. Then there exists a solution $\hat{\theta}$ of $\partial L / \partial \theta=0$ which gives a consistent estimate of $\theta^{0}$. If $y(t)$ is a random vector with components

$$
y_{u}=n^{-\frac{1}{2}} \sum_{k=1}^{n} G_{u}\left(\xi_{k}, \xi_{k+1}, \beta_{k+1}, \theta^{0}\right), \quad u=1, \ldots, r
$$

and $l(t)$ is the random vector with components $l_{u}(t)=n^{\frac{1}{2}}\left(\hat{\theta}_{u}-\theta_{u}^{0}\right), \quad u=1, \ldots, r$ then as $n \rightarrow \infty$ $y(t) \stackrel{\mathcal{L}}{\rightarrow} \mathcal{N}\left(0, \sigma\left(\theta^{0}\right)\right), l(t) \stackrel{\mathcal{L}}{\rightarrow} \mathcal{N}\left(0, \sigma\left(\theta^{0}\right)^{-1}\right), 2\left[L(\hat{\theta})-L\left(\theta^{0}\right)\right] \stackrel{\mathcal{L}}{\rightarrow} \chi_{r}^{2}$

We check that the conditions of the above theorem are satisfied in the present situation.

$\diamond$ Condition (1). $\bar{\lambda}_{2}$ is bounded, therefore condition 1 is easily checked.

$\diamond$ Condition (2). It is easily checked because for every $\theta \in \Theta$ the $\{\eta ; f(\xi, \eta, \theta)>0\}=\mathbb{R}_{+}$. 
$\diamond$ Condition (3). We can rewrite the function $F(\xi, \beta, \eta, \theta)$ as a function of only the parameter vector $\theta$ as follows:

$$
F(\theta)= \begin{cases}\bar{\lambda}_{2}(\xi) e^{-\lambda_{d} \xi} e^{-\left(\lambda_{1}+\bar{\lambda}_{2}(\xi)\right) \beta} ; & \eta=0 \\ \bar{\lambda}_{2}(\xi) \lambda_{d} e^{-\lambda_{d}(\xi-\eta)} e^{-\left(\lambda_{1}+\bar{\lambda}_{2}(\xi)\right) \beta} ; & 0<\eta<\xi \\ \lambda_{1} \lambda_{u} e^{-\lambda_{u}(\eta-\xi)} e^{-\left(\lambda_{1}+\bar{\lambda}_{2}(\xi)\right) \beta} ; & \xi \leq \eta\end{cases}
$$

Now it is easy to see that $F$ possesses partial derivatives of order three because $\bar{\lambda}_{2}$ does.

$\diamond$ Condition (4). The first two integrability conditions are easy to see from the expression (12). To obtain the required integrability we need $F_{u}\left(F_{u v}\right)$ to be bounded in $\eta$ for $\eta<\xi$ and integrable in for $\eta \geq \xi$. (We can first integrate w.r. to $\beta$.) Boundedness is apparent because of continuity of the derivatives. The integrability follows from the fact that for $\eta \geq \xi$ the $F_{u}\left(F_{u v}\right)$ can be written as: $F_{u}(\eta, \beta)=h(\eta, \beta) e^{-a \eta-b \beta}$ where $a, b>0$ and $h(\eta, \beta)$ is $O\left(\eta^{n} \beta^{m}\right)$ for some integers $n, m$. To check the finiteness of the expectation we prove more, namely that $G_{u v w}$ is bounded almost surely. $G$ can be written as:

$$
G(\xi, \beta, \eta, \theta)= \begin{cases}\ln \bar{\lambda}_{2}(\xi)-\lambda_{d} \xi-\left(\lambda_{1}+\bar{\lambda}_{2}(\xi)\right) \beta ; & \eta=0 \\ \ln \bar{\lambda}_{2}(\xi)+\ln \lambda_{d}-\lambda_{d}(\xi-\eta)-\left(\lambda_{1}+\bar{\lambda}_{2}(\xi)\right) \beta ; & 0<\eta<\xi \\ \ln \lambda_{1}+\ln \lambda_{u}-\lambda_{u}(\eta-\xi)-\left(\lambda_{1}+\bar{\lambda}_{2}(\xi)\right) \beta ; & \xi \leq \eta\end{cases}
$$

It is clear that only very few of the third order derivatives are non-zero. Namely $G_{\lambda_{1}^{3}}=\frac{2}{\lambda_{1}^{3}} 1_{\{\eta \geq \xi\}}$, $G_{\lambda_{d}^{3}}=\frac{2}{\lambda_{d}^{3}} 1_{\{0<\eta<\xi\}}, G_{\lambda_{u}^{3}}=\frac{2}{\lambda_{u}^{3}} 1_{\{\eta \geq \xi\}}$ and mixed derivatives with respect to $\lambda_{2}^{(I)}$ and $\lambda_{2}^{(I I)}$ for $0<\eta<\xi$. We will denote $G_{i j}=G_{\left(\lambda_{2}^{(I)}\right)^{i}\left(\lambda_{2}^{(I I)}\right)^{j}}$. Then $G_{21}=\frac{2 \kappa(\xi)}{\lambda_{2}(\xi)^{3}}, G_{30}=\frac{2}{\lambda_{2}(\xi)^{3}}, G_{12}=\frac{2 \kappa(\xi)^{2}}{\lambda_{2}(\xi)^{3}}$, $G_{03}=\frac{2 \kappa(\xi)^{3}}{\lambda_{2}(\xi)^{3}}$. Since the former are constant and the latter are bounded for all $\xi, \eta, \beta$ they satisfy the condition 3 .

$\diamond$ Condition (5) is satisfied as long as the following conditions hold true for some $\delta>0$

$$
\begin{aligned}
\mathbb{E}_{\theta}\left[\left|\rho_{2}\right|^{2+\delta}\right] & <\infty, \\
\mathbb{E}_{\theta}\left[\left|\rho_{2} z_{1}\right|^{2+\delta}\right] & <\infty,
\end{aligned}
$$

as can be seen from the special form of the partial derivatives of $G$ :

$$
\begin{aligned}
G_{\lambda_{1}} & =\left\{\begin{array}{rr}
-\beta & \eta<\xi \\
\frac{1}{\lambda_{1}}-\beta & \eta \geq \xi
\end{array}, \quad G_{\lambda_{d}}=\left\{\begin{array}{rr}
\frac{1}{\lambda_{d}}-(\xi-\eta) & \begin{array}{r}
-\xi \\
0
\end{array} \\
0 & \eta \geq \xi
\end{array},\right.\right. \\
G_{\lambda_{2}^{(I I)}} & =\left\{\begin{array}{rr}
\frac{\kappa(\xi)}{\lambda_{2}(\xi)}-\kappa(\xi) \beta \\
-\kappa(\xi) \beta & \eta \geq \xi
\end{array}, \quad G_{\lambda_{u}}=\left\{\begin{array}{rr}
0 & \eta<\xi \\
\frac{1}{\lambda_{u}}-(\eta-\xi) & \eta \geq \xi
\end{array}\right.\right. \\
G_{\lambda_{2}^{(I)}} & =\left\{\begin{array}{rr}
\frac{1}{\lambda_{2}(\xi)}-\beta & \eta<\xi \\
-\beta 1_{\{\xi>0\}} & \eta \geq \xi
\end{array}\right.
\end{aligned}
$$


We first prove (14):

$$
\begin{aligned}
\mathbb{E}_{\theta}\left[\left|\rho_{2}\right|^{(2+\delta)}\right] & =\int \mathbb{E}_{\theta}\left[\left|\rho_{2}\right|^{(2+\delta)} \mid z_{1}=z\right] \pi_{\theta}(d z) \\
& \leq \int \mathbb{E}_{\theta}\left[\left|\rho_{2}\right|^{(2+\delta)} \mid z_{1}=0\right] \pi_{\theta}(d z) \\
& =\mathbb{E}_{\theta}\left[\Lambda_{1}^{(2+\delta)}\right]<\infty
\end{aligned}
$$

where $\Lambda_{1}$ is an exponential random variable with parameter $\lambda_{1}$. To prove (15) we notice that:

$$
\begin{aligned}
\mathbb{E}_{\theta}\left[\left|z_{1} \rho_{2}\right|^{(2+\delta)}\right] & =\int \mathbb{E}_{\theta}\left[\left|z \rho_{2}\right|^{(2+\delta)} \mid z_{1}=z\right] \pi_{\theta}(d z) \\
& \geq \int \mathbb{E}_{\theta}\left[\left|z \Lambda_{M}\right|^{(2+\delta)} \mid z_{1}=z\right] \pi_{\theta}(d z) \\
& =\mathbb{E}_{\theta}\left[\Lambda_{M}^{(2+\delta)}\right] \mathbb{E}_{\theta}\left[z_{1}^{(2+\delta)}\right]
\end{aligned}
$$

where $\Lambda_{M}$ is an exponential random variable with parameter $\lambda_{1}+M$ independent of $z_{1}$. Similarly we can show:

$$
\mathbb{E}_{\theta}\left[\left|z_{1} \rho_{2}\right|^{(2+\delta)}\right] \leq \mathbb{E}_{\theta}\left[\Lambda_{1}^{(2+\delta)}\right] \mathbb{E}_{\theta}\left[z_{1}^{(2+\delta)}\right]
$$

Therefore it is enough (and necessary) to prove that $\mathbb{E}_{\theta}\left[\left|z_{1}\right|^{(2+\delta)}\right]<\infty$. That however, follows from equation (19) of Theorem 2.7 coupled with the drift condition (16) below. Since those imply that the stationary distribution $\pi_{\theta}$ possesses all moments.

We proceed to prove the non-singularity of the matrix $\sigma$. First we notice that

$$
\sigma_{u v}=\mathbb{E}\left[G_{u} G_{v}\right]=-\mathbb{E}\left[G_{u v}\right] .
$$

In this form it is easy to see that the matrix $\sigma$ has only two non-zero off diagonal elements, namely

$$
-\mathbb{E}\left[G_{\lambda_{2}^{(I I)} \lambda_{2}^{(I)}}\right]=-\mathbb{E}\left[G_{\lambda_{2}^{(I)} \lambda_{2}^{(I I)}}\right]=\mathbb{E}\left[1_{\{\xi>0\}} \frac{\kappa(\xi)}{\bar{\lambda}_{2}^{2}(\xi)}\right] .
$$

Therefore $\sigma$ is non-singular if and only if the matrix:

$$
\left(\begin{array}{cc}
\mathbb{E}\left[1_{\{\xi>0\}} \frac{\kappa(\xi)^{2}}{\lambda_{2}^{2}(\xi)}\right] & \mathbb{E}\left[1_{\{\xi>0\}} \frac{\kappa(\xi)}{\lambda_{2}^{2}(\xi)}\right] \\
\mathbb{E}\left[1_{\{\xi>0\}} \frac{\kappa(\xi)}{\lambda_{2}^{2}(\xi)}\right] & \mathbb{E}\left[1_{\{\xi>0\}} \frac{1}{\lambda_{2}^{2}(\xi)}\right]
\end{array}\right)
$$

is non-singular. This is true since its determinant is positive for all values of $\lambda_{2}^{(I)}, \lambda_{2}^{(I I)}$.

$\diamond$ Condition (6). We shall prove this condition in a series of statements. The main idea will be to show that there exists a "small" subset of the state space towards which the Markov chain $\xi$ has a geometric drift. Because of Theorem 2.7, this implies the existence of a unique stationary distribution of $\xi$. But first we recall a couple of standard definitions for the sake of definiteness.

Definition 2.3. Let $P$ be the transition kernel of a Markov chain on a measurable space $(X, \mathcal{B}(X))$. A set $C \in \mathcal{B}(X)$ is called small if there exists an $m>0$, and a non-trivial measure $\nu_{m}$ on $\mathcal{B}(X)$, such that for all $x \in C, B \in \mathcal{B}(X) P^{m}(x, B) \geq \nu_{m}(B)$.

Definition 2.4. The Markov chain $\xi$ is called strongly aperiodic if there exists a $\nu_{1}$-small set $A$ such that $\nu_{1}(A)>0$. Moreover, the chain is said to be $\phi$-irreducible if for all $x \in X$ and any $A \in \mathcal{B}(X)$ such that $\phi(A)>0, L(x, A)>0$ where $L(x, A)$ is the probability of reaching $A$ starting from $x$. 
Proposition 2.5. Any interval $\left[0, x_{0}\right]$ is a small set for the chain $\xi$.

Proof. We first we show that $\{0\}$ is a small set. Let $m=1$ and $\nu=P^{\xi}(0, d y)=f(0, y)\left(d y+\delta_{0}\right)$. By definition for any $A \in \mathcal{B}(\mathcal{R}), P^{\xi}(\{0\}, A)=\nu$. For any $x \in\left[0, x_{0}\right], P^{\xi}(x,\{0\})>0$ therefore by Proposition 5.2.4 [?] the interval $\left[0, x_{0}\right]$ is a small set too.

Proposition 2.6. There exists $x_{0} \geq 0$ such that the Markov chain $\xi$ has a geometric drift towards $\left[0, x_{0}\right]$ i.e. there exists $\beta>0$ and $b<\infty$ such that for any $x>0$ :

$$
\int P^{\xi}(x, d y) V(y) \leq(1-\beta) V(x)+b 1_{\left[0, x_{0}\right]}(x)
$$

where $V(x)=e^{\gamma x}, 0<\gamma<\lambda_{u}$.

Proof. Let us denote $p(x)=\frac{\lambda_{1}}{\lambda_{1}+\bar{\lambda}_{2}(x)}, q(x)=\frac{\bar{\lambda}_{2}(x)}{\lambda_{1}+\bar{\lambda}_{2}(x)}$ We have:

$$
\begin{aligned}
P^{\xi} V & =\int_{0}^{\infty} P^{\xi}(x, d y) V(y)= \\
& =p(x) \int_{x}^{\infty} \lambda_{u} e^{-\lambda_{u}(y-x)} e^{\gamma y} d y+q(x)\left[\int_{0}^{x} \lambda_{d} e^{-\lambda_{d}(x-y)} e^{\gamma y} d y+e^{-\lambda_{d} x}\right] \\
& =p(x) \frac{\lambda_{u}}{\lambda_{u}-\gamma} e^{\gamma x}+q(x)\left[\frac{\lambda_{d}}{\lambda_{d}+\gamma}+e^{-x\left(\lambda_{d}+\gamma\right)}\left(1-\frac{\lambda_{d}}{\lambda_{d}+\gamma}\right)\right]
\end{aligned}
$$

Let us denote $D(\gamma)=\frac{\lambda_{d}}{\lambda_{d}+\gamma}, U(\gamma)=\frac{\lambda_{u}}{\lambda_{u}-\gamma}$. Recall that $0<\gamma<\lambda_{u}$. Rearranging (17) we obtain $P^{\xi} V=e^{\gamma x}\left\{p(x) U(\gamma)+q(x)\left[D(\gamma)+e^{-x\left(\lambda_{d}+\gamma\right)}(1-D(\gamma))\right]\right\}$. Denoting $A(x)=D(\gamma)+$ $e^{-x\left(\lambda_{d}+\gamma\right)}(1-D(\gamma))$ we get:

$$
P^{\xi} V=V(x)(p(x) U(\gamma)+q(x) A(x))=V(x)[p(x)(U(\gamma)-A(x))+A(x)]
$$

Let us define $B(x)=\frac{1-A(x)}{U \gamma-A(x)}$. $B(x)$ is well defined for all $x \geq 0$ because $0<A(x) \leq 1$ and $U(\gamma)>1$. Taking derivative we get:

$$
B^{\prime}(x)=A^{\prime}(x)(1-U(\gamma))=-\left(\lambda_{d}+\gamma\right) e^{-x\left(\lambda_{d}+\gamma\right.}(1-D(\gamma))(1-U(\gamma))>0 .
$$

Thus $B(x)$ is an increasing function of $x$. It is easy to see that $p(x)$ is a decreasing function of $x$ as long as $\bar{\lambda}_{2}(x)$ is increasing. Now:

$$
B(\gamma)=\lim _{x \rightarrow \infty} B(x)=\frac{1-D(\gamma)}{U(\gamma)-D(\gamma)}
$$

and

$$
p(M)=\lim _{x \rightarrow \infty} p(x)=\frac{\lambda_{1}}{\lambda_{1}+M} .
$$

For any $0<\gamma<\lambda_{u}$ we can choose $M$ large enough so that there is $\beta_{1}>0$ such that $p(M)<$ $B(\gamma)-\beta_{1}$. For any $0<\beta<\beta_{1}$ we can then define $x_{0}=$ inf $H, \quad H=\{x \geq 0: p(x)<B(x)-\beta\}$. $H$ is non-empty and bounded from below by 0 so we have $x_{0} \geq>0$. For $x>x_{0}$ we have in (18) $P^{\xi} V(x)<V(x)(1-\beta)$, therefore the drift condition (16) holds. To show that it also holds for $0 \leq x \leq x_{0}$, we only need that $\left[P^{\xi} V\right](x)$ is bounded there, and this is easy to see from (18). 
Theorem 2.7 (Theorem 15.0.1 [?]). Suppose that the chain $\xi$ is $\psi$-irreducible and strongly aperiodic. Then the following conditions are equivalent:

(1) The chain is positive recurrent with invariant probability measure $\pi$, and there exists some $\nu$-small set $C$ with $\psi(C)>0, \rho_{C}<1$ and $M_{C}<\infty$, and $P^{\infty}(C)>0$ such that for all $x \in C:\left|P^{n}(x, C)-P^{\infty}(C)\right| \leq M_{C} \rho_{C}^{n}$.

(2) There exists some small set $C$ with $\psi(C)>0$ and $\kappa>1$ such that $\sup _{x \in C} E_{x}\left[\kappa^{\tau_{C}}\right]<\infty$

(3) There exists a small set $C$, constants $b<\infty, \beta>0$ and a function $V \geq 1$ finite at some $x_{0} \in X$ satisfying $P^{\xi} V(x) \leq(1-\beta) V(x)+b 1_{C}(x), \quad x \in X$

Any of the three conditions imply that the set $S_{V}=\{x: V(x)<\infty\}$ satisfies $P\left(x, S_{V}\right)=1$ for all $x \in S_{V}$. Hence, there exist constants $r>1, R<\infty$ such that for any $x \in S_{V}$

$$
\sum_{n} r^{n}\left\|P^{n}(x, \cdot)-\pi\right\| \leq R V(x)
$$

Since the Markov chain $\xi$ satisfies the general conditions of Theorem 2.7, and since condition (3) is clearly satisfied, then (1) gives the existence of a unique stationary distribution $\pi_{\theta}$ for each $\theta \in \Theta$.

Proposition 2.8. For any $x \in X, \pi_{\theta}(x, \cdot) \ll \pi_{\theta}(\cdot)$

Proof. To prove the statement we only need to show that the measure $\mu=d y+\delta_{0}$ on $\mathbb{R}^{+}$is absolutely continuous with respect to $\pi_{\theta}$ since $K$ has density $f$ with respect to $\mu$.

First we show that $\pi_{\theta}(\{0\})>0$. Clearly, there is a set $B \in \mathcal{B}(X)$ such that $\pi_{\theta}(B)>0$. Let $x_{B}=\inf B<\infty$. Then

$$
\pi_{\theta}(\{0\}) \geq \int_{B} \pi_{\theta}(d x) \pi_{\theta}(x,\{0\}) \geq \int_{B} \pi_{\theta}(d x) e^{-\lambda_{d} x_{B}} \geq e^{-\lambda_{d} x_{B}} \pi_{\theta}(B)>0 .
$$

$\mathrm{iF}[a, b)$ is an arbitrary semi-open interval contained in $\mathbb{R}_{+}$then

$$
\pi_{\theta}([a, b)) \geq \pi_{\theta}(\{0\}) \int_{a}^{b} \lambda_{u} e^{-\lambda_{u} y} d y>0 .
$$

Corollary 2.9. If the first order conditions have a unique solution $\hat{\theta}$, and if the conditions of Theorem 2.2 are satisfied, then $\hat{\theta}$ is a consistent MLE estimate of the true parameter $\theta^{0}$.

It is clear from the computations preceding Theorem (2.2) that $\lambda_{1}, \lambda_{u}$ and $\lambda_{d}$ are uniquely determined. Proposition 2.1 shows the uniqueness for the other parameters. One remaining open question is whether the system (4) and (5) has a solution at all. It is addressed in the following proposition.

Proposition 2.10. With probability one, for $n$ large enough, the system of equations (4) and (5) has a solution.

Proof. To prove the statement we show that $\lim _{n \rightarrow \infty} g(0)>0$ almost surely. Since

$$
\begin{aligned}
g(0) & =\frac{B}{|D|} \sum_{i \in D} \kappa\left(\xi_{i}\right)-C \\
& =\left(\sum_{i \in D} \beta_{i+1} \frac{1}{|D|} \sum_{i \in D} \kappa\left(\xi_{i}\right)-\sum_{i \in D} \kappa\left(\xi_{i}\right) \beta_{i+1}\right)+\left(\sum_{i \in U} \beta_{i+1} \frac{1}{|D|} \sum_{i \in D} \kappa\left(\xi_{i}\right)-\sum_{i \in U} \kappa\left(\xi_{i}\right) \beta_{i+1}\right),
\end{aligned}
$$


then the law of large numbers implies that:

$$
\begin{aligned}
& \lim _{n \rightarrow \infty} \frac{1}{|D|}\left(\sum_{i \in D} \beta_{i+1} \frac{1}{|D|} \sum_{i \in D} \kappa\left(\xi_{i}\right)-\sum_{i \in D} \kappa\left(\xi_{i}\right) \beta_{i+1}\right) \\
& =\mathbb{E}\left(\rho_{2} \mid z_{1}, z_{1}-z_{2}>0\right) E\left(\kappa\left(z_{1}\right) \mid z_{1}-z_{2}>0\right)-\mathbb{E}\left(\rho_{2} \mid z_{1}, z_{1}-z_{2}>0\right) \mathbb{E}\left(\kappa\left(z_{1}\right) \mid z_{1}-z_{2}>0\right)=0
\end{aligned}
$$

and

$$
\begin{aligned}
& \lim _{n \rightarrow \infty} \frac{1}{|U|}\left(\sum_{i \in U} \beta_{i+1} \frac{1}{|D|} \sum_{i \in D} \kappa\left(\xi_{i}\right)-\sum_{i \in U} \kappa\left(\xi_{i}\right) \beta_{i+1}\right) \\
& =\mathbb{E}\left(\rho_{2} \mid z_{1}, z_{1}-z_{2}<0\right) E\left(\kappa\left(z_{1}\right) \mid z_{1}-z_{2}>0\right)-\mathbb{E}\left(\rho_{2} \mid z_{1}, z_{1}-z_{2}<0\right) E\left(\kappa\left(z_{1}\right) \mid z_{1}-z_{2}<0\right)>0 .
\end{aligned}
$$

Combining the two we get:

$$
\lim _{n \rightarrow \infty} g(0)=\infty>0 .
$$

By this we have established that the MLE estimator which we derived, possesses all the properties stated in theorem 2.2.

As mentioned above to get a complete rainfall intensity model the temporal dynamics of storms' arrivals and durations has to be specified. The inspiration can be taken from the BLPC model which assumes the storms arrive according to a Poisson process and last for a random (exponentially distributed) time; they can overlap. In the framework of the proposed model the random number of storms active at each moment manifests itself in the random cell arrival rate. This can be captured by replacing the constant cell arrival rate parameter $\lambda_{1}$ by a stochastic process $\bar{\lambda}_{1}$. If the dynamics of the rainfall process conditional on the realization of stochastic process $\bar{\lambda}_{1}$ is given by (1) then MLE can be performed separately for parameters of the dynamics of $\bar{\lambda}_{1}$. This gives us freedom to choose a model for $\bar{\lambda}_{1}$ for which a valid estimation procedure (preferably MLE) is feasible.

A simple model whose MLE is well established is that of switching Markov process for $\bar{\lambda}_{1}$. The state space consist of two states $\left\{0, \lambda_{1}\right\}$ with transition rates from states $q_{d}, q_{p}$. The ML estimators for $\lambda_{1}, q_{d}, q_{p}$ in this model have been presented in [?]. Assuming one observes the inter-arrival times $U=\left(U_{1}, U_{2}, \ldots, U_{n}\right)$ of a Cox process $N$ directed by a switching Markov process $M$, the likelihood function can be shown to have the following form:

$$
\begin{aligned}
L\left(U, \lambda_{1}, q_{d}, q_{p}\right) & =\prod_{i=1}^{n} f\left(U_{i}, \lambda_{1}, q_{d}, q_{p}\right) \\
f\left(x, \lambda_{1}, q_{d}, q_{p}\right) & =\frac{1}{r_{2}-r_{1}}\left[\left(q_{d}+q_{p}-r_{1}\right) r_{1} e^{-r_{1} x}-\left(q_{d}+q_{p}-r_{2}\right) r_{2} e^{-r_{2} x}\right] \\
r_{1} & =\frac{1}{2}\left(q_{d}+q_{p}+\lambda_{1}\right)-\sqrt{\frac{1}{4}\left(q_{d}+q_{p}+\lambda_{1}\right)^{2}-l_{1} q_{d}} \\
r_{2} & =\frac{1}{2}\left(q_{d}+q_{p}+\lambda_{1}\right)+\sqrt{\frac{1}{4}\left(q_{d}+q_{p}+\lambda_{1}\right)^{2}-l_{1} q_{d}}
\end{aligned}
$$


Once the parameters of $\bar{\lambda}_{1}$ are estimated, the MLE method for estimating the remaining parameters described above can be used, due to the separation properties of the likelihood function mentioned above.

2.4. Comparison with Rodriguez-Iturbe's Model. In this section we check numerically that our Markov jump model (MJ) leads to properties quite analog to those of the widely accepted Poisson pulse (PP) model of Rodriguez-Iturbe.

We choose three main characteristics of the precipitation to compare the two models: duration of dry spells, duration of wet spells, and accumulated rainfall volume during a single wet spell. We perform the comparison at the level of a single storm because that is where the models differ. We show that the probability distributions of these quantities are very similar in both MJ and PP models. These distributions are not explicitly known either for the PP model ([?]) or for our MJ model, therefore we rely on Monte Carlo simulations to generate samples from the two models and compare the resulting empirical distributions of the relevant characteristics.

In order to set up a reasonable comparison, we have to resolve the issue of the different parameter sets driving the two models. This calibration can easily be done because the three parameters of Rodriguez-Iturbe model $\lambda$ - cells arrival rate, $\mu_{x}$ - average cell intensity and $\eta$ - cell extinction rate can be naturally linked to the jump Markov model in a nearly one-to-one fashion as follows: $\lambda_{1}=\lambda$, $1 / \lambda_{u}=1 / \lambda_{d}=\mu_{x}, \lambda_{2}^{(I I)}=\eta$.

Although the choice of exact parameter values is not important for our comparison, to obtain realistic values for $\lambda, \mu_{x}$ and $\eta$, we fit PP model to NY Westchester January data using a form of the method of moments suggested in [?]. Based on that, we set: $\lambda=180, \mu_{x}=1500, \eta=400$ and generate 360 months worth of precipitation data from each model. We set the extra parameter $\lambda_{2}^{(I)}=300$ so that the empirical average wet spell length in both models coincide. Samples from
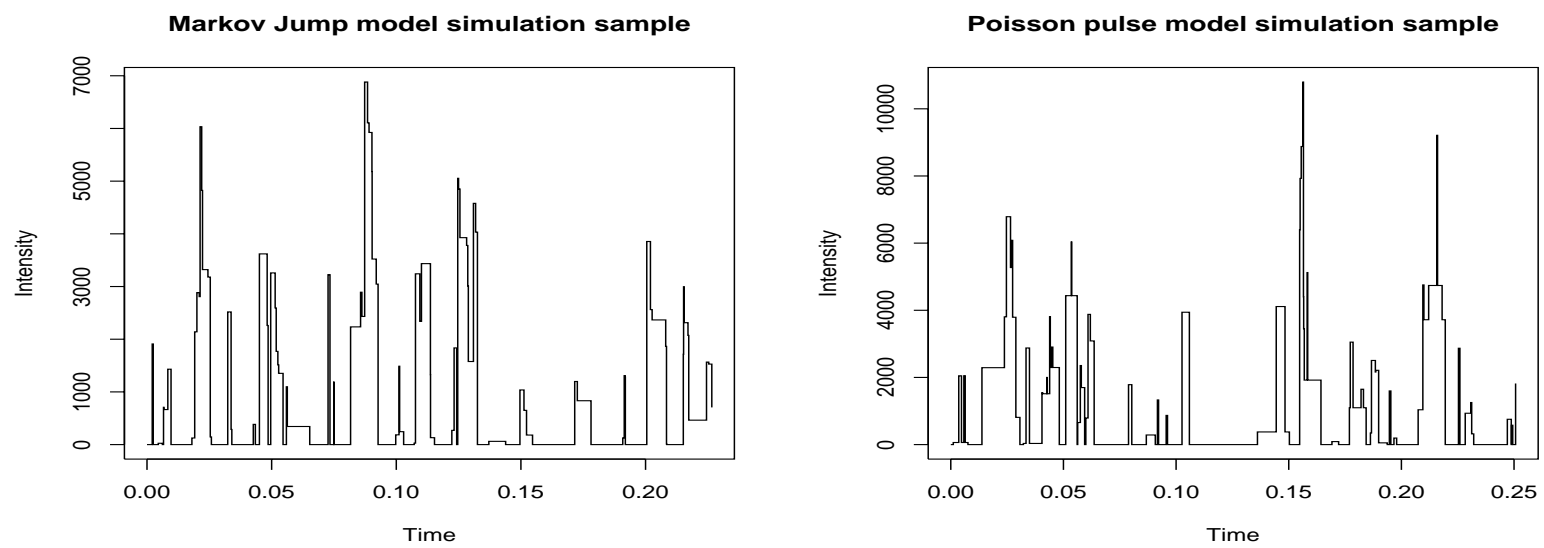

Figure 1. Monte Carlo samples from the Markov jump model (left) and from the Poisson pulse model (right).

both models are shown in Figure 1 for the purpose of illustration. There is no obvious difference in the statistics of the precipitation patterns they produce. As evidenced by the Q-Q plot in the center 

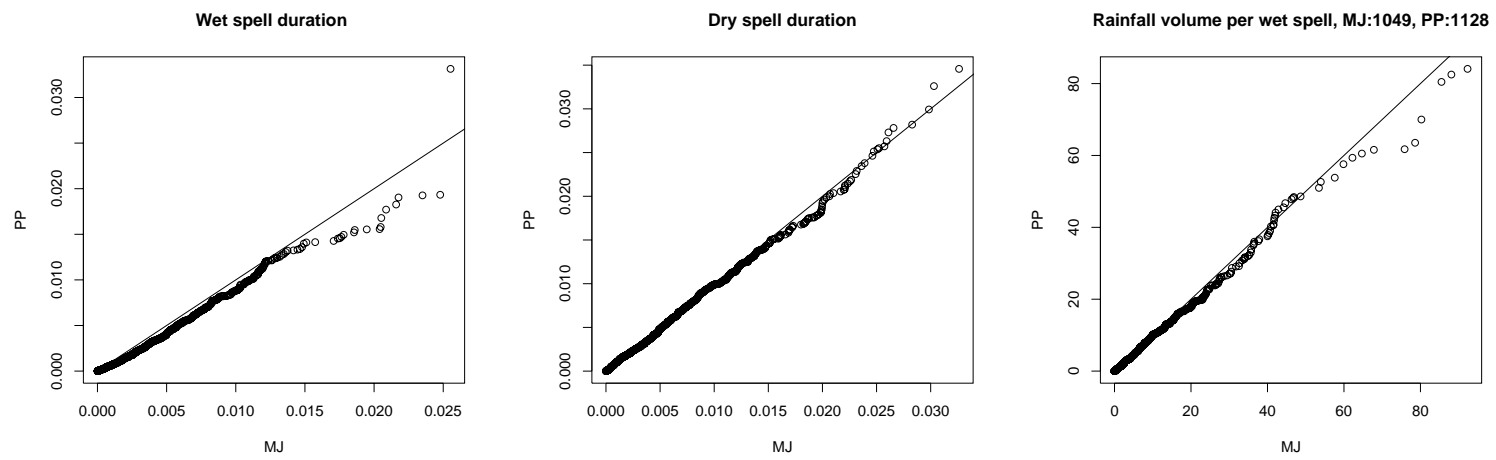

FIGURE 2. Q-Q plots of the wet (left) and dry (right) spell durations from the Markov jump and Poisson pulse models.

pane of Figure 2, the distributions of the lengths of the dry spells seem to coincide in both models. The Q-Q plot in the right pane shows that the distributions of the rainfall volumes also agree in the two models, though to a lesser extend in the case of the simulations at hand. Finally, the Q-Q plot in the left pane shows that, at least in the case of the Monte Carlo samples which we generated, the distribution of the length of a typical wet spell could have heavier tail in the Markov jump model. However, the bulk of the data seems to be in a region where the two distributions agree.

\section{Pricing Precipitation Options}

3.1. Security Price as Random Evolution. We generalize the standard geometric Brownian motion security price model by allowing the coefficients $\mu$ and $\sigma$ to depend on another process $Y$. Formally we assume that the dynamics of the traded asset $S$ are given by a stochastic differential equation of the form:

$$
d S_{t}=S_{t}\left(\mu\left(Y_{t}\right) d t+\sigma\left(Y_{t}\right) d W_{t}\right)
$$

where the process $Y=\left\{Y_{t}\right\}$ is a continuous time Markov process in $\mathbb{R}^{n}$ independent of the Wiener process $W$. Models of this form are common in financial applications: regime switching models, stochastic volatility models. Also, this is the model used in [?] to price temperature options. The conditions for the existence and uniqueness of the solution to (20) are discussed in [?]. The relevant results for our purposes are stated in Theorem 3.1 and its corollary. The pair $\left(S_{t}, Y_{t}\right)$ forms a Markov process which is known in the literature as random evolution.

Theorem 3.1. Let there exist a constant $K$ and real functions $g(t, y), h(t, y)$ such that:

$$
\begin{aligned}
\left|\mu\left(t, x_{1}, y\right)-\mu\left(t, x_{2}, y\right)\right| & \leq K\left|x_{1}-x_{2}\right| \\
\left|\sigma\left(t, x_{1}, y\right)-\sigma\left(t, x_{2}, y\right)\right| & \leq K^{2}\left|x_{1}-x_{2}\right| \\
\frac{1}{2}|\sigma(t, x, y)|^{2} & \leq g(t, y)^{2}+K^{2}|x|^{2} \\
|\mu(t, x, y)| & \leq h(t, y)+K^{2}|x|
\end{aligned}
$$


and for all $m=1,2,3, \ldots: \mathbb{E}\left[\int_{0}^{T}\left(g\left(s, Y_{s}\right)^{m}+h\left(s, Y_{s}\right)^{m}\right) d s\right]<\infty$ then (20) with the initial data $X_{0}=x$ has a pathwise unique solution and it is $\mathcal{F}_{t}$-progressively measurable.

Corollary 3.2. If $Y$ is real valued and for every $m>0$ :

$$
\mathbb{E}\left[\int_{0}^{T}\left|Y_{s}\right|^{m} d s\right]<\infty
$$

then conditions (22) are equivalent to:

$$
|\mu(t, x, y)| \leq C(|x|+|y|)^{m}, \quad \text { and } \quad|\sigma(t, x, y)| \leq C(|x|+|y|)^{m} .
$$

3.2. Utility Maximization as a Stochastic Control Problem. We now formalize the problem described in the introduction. We are given a filtered probability space $\left(\Omega, \mathcal{F},\left\{\mathcal{F}_{t}\right\}_{0<t<T}, P\right) . \mathcal{F}_{t}$ is the sigma field $\sigma\left(\left(S_{s}, Y_{s}\right): 0 \leq s \leq t\right)$ augmented by the null-sets of $\mathcal{F}=\mathcal{F}_{T}$. Trading is allowed in a risky asset whose price process is a random evolution $\left(S_{t}, Y_{t}\right)$ defined in Subsection 3.1 and a bank account for which we assume that the interest rate is zero, and we assume that the agent possesses initial wealth $x$. We look for a self financing trading strategy $\phi$ - the amount of money invested in the risky asset - that maximizes the utility of the terminal wealth. For any strategy, the dynamics of the wealth process are given by:

$$
d X_{t}=\phi_{t}\left(\mu\left(Y_{t}\right) d t+\sigma\left(Y_{t}\right) d W_{t}\right)
$$

and the objective is to maximize:

$$
\mathbb{E}\left[U\left(X_{T}\right)\right]
$$

Clearly, for any admissible strategy, the pair $(X, Y)$ also forms a random evolution. The problem of maximizing (24) for the process (23) above falls in the framework of controlled Markov processes. As such, it can be solved by dynamic programming, and the conditions for the existence of the optimal solution are given in verification theorems [?].

3.3. HJB Equation for Random Evolutions. Candidates for the solution of problem (24) among Markovian controls can be found by solving the corresponding HJB equation. The controlled backward evolution operator $A^{u}$ of the random evolution $(X, Y)$ reads:

$$
A^{u} V(t, x, y)=\frac{\partial V}{\partial t}+u \mu(y) \frac{\partial V}{\partial x}+\frac{1}{2} u^{2} \sigma^{2}(y) \frac{\partial^{2} V}{\partial x^{2}}+G V(t, x, y)
$$

where $G$ is the infinitesimal generator of the process $Y$. Hence the HJB equation for the value function $V$ of the optimization problem (24) is:

$$
0=\sup _{u} A^{u} V=\frac{\partial V}{\partial t}+G V+\sup _{u}\left\{u \mu(y) \frac{\partial V}{\partial x}+\frac{1}{2} u^{2} \sigma^{2}(y) \frac{\partial^{2} V}{\partial x^{2}}\right\} .
$$

We can perform the maximization with respect to $u$ explicitly to get the optimal control $u^{*}$ and an integro-differential HJB equation: 


$$
\begin{gathered}
u^{*}=-\frac{\mu(y)}{\sigma^{2}(y)} \frac{\partial V / \partial x}{\partial^{2} V / \partial x^{2}} \\
0=\frac{\partial V}{\partial t}+G V-\frac{1}{2} \frac{\mu^{2}(y)}{\sigma^{2}(y)} \frac{(\partial V / \partial x)^{2}}{\partial^{2} V / \partial x^{2}} .
\end{gathered}
$$

The terminal condition for the backward HJB equation (28) is provided by by the utility function $U$, namely: $V(T, x, y)=U(x)$. If we assume the process $Y$ is the jump Markov process storm model developed in section (2.2) we obtain in particular:

$$
0=\frac{\partial V}{\partial t}+\lambda(y) \int_{R}[V(t, x, z)-V(t, x, y)] \Pi(y, d z)-\frac{1}{2} \frac{\mu^{2}(y)}{\sigma^{2}(y)} \frac{(\partial V / \partial x)^{2}}{\partial^{2} V / \partial x^{2}}
$$

where $\lambda$ is the jump rate function and $\Pi$ is the jump transition kernel for the process $Y$.

In order for a solution $V$ of the HJB equation to be the value function of the stochastic control problem it needs to satisfy the conditions of the verification theorem [?]. In particular it needs to belong to $\mathcal{D}$ the common domain of the backward evolution operators $A^{u} ; u \in U$. Before we proceed to obtain explicit solutions to (28) we characterize the set $\mathcal{D}$ for the equation (29).

Proposition 3.3. The domain $\mathcal{D}$ of the HJB equation (29) contains all continuous functions $\Psi(t, x, y)$ defined on $[0, T] \times \mathbb{R}^{2}$, such that:

(1) $\partial \Psi / \partial t, \partial \Psi / \partial x, \partial^{2} \Psi / \partial x^{2}$ are continuous.

(2) $\Psi, \partial \Psi / \partial t, \partial \Psi / \partial x, \partial^{2} \Psi / \partial x^{2}$ have polynomial growth, i.e. for some $K$ and $m$ :

$$
|\Psi(t, x, y)|+|\partial \Psi / \partial t(t, x, y)|+|\partial \Psi / \partial x(t, x, y)|+\left|\partial^{2} \Psi / \partial x^{2}(t, x, y)\right| \leq K(1+|x|+|y|)^{m}
$$

Proof. We will show that for all $u \in U$ any $\Psi$ satisfying the conditions of the proposition belongs to the domain $\mathcal{D}\left(A^{u}\right)$ of the operator $A^{u}$ (along the lines of [?], Appendix B). Let us fix an arbitrary $u \in U$. For all $\Psi$ satisfying the conditions 1 and 2 the operator $A^{u}$ is well defined. By condition 1 all $\Psi, \partial \Psi / \partial t$ and $A \Psi$ are continuous functions. Condition 2 guarantees that for any $s>t$ : $\mathbb{E}_{t x}\left|\Psi\left(s, X_{s}, Y_{s}\right)\right|<\infty$ and $\int_{t}^{s}\left|A \Psi\left(r, X_{r}, Y_{r}\right)\right|<\infty$. It remains to show that such functions satisfy the Dynkin formula $\mathbb{E}_{t x y}\left[\Psi\left(s, X_{s}, Y_{s}\right)\right]-\Psi(t, x, y)=\mathbb{E}_{t x y}\left[\int_{t}^{s} A^{u} \Psi\left(r, X_{r}, Y_{r}\right) d r\right] ; \quad t<s \leq T$

We can decompose the operator $A^{u}$ in two parts $A^{u} \Psi=A_{0}^{u} \Psi+H \Psi$ where:

$$
\begin{aligned}
A_{0}^{u} \Psi(t, x, y) & =\frac{\partial \Psi}{\partial t} \mu(y) \frac{\partial}{\partial} x f+\frac{1}{2} \sigma^{2}(y) \frac{\partial^{2}}{\partial x^{2}} f \\
H \Psi(t, x, y) & =\int_{R}[\Psi(t, x, z)-\Psi(t, x, y)] \Pi(y, d z)
\end{aligned}
$$

Let $t_{1}<t_{2}<\cdots<t_{N}$ be the jump times of the process $Y$ on [t,s] and let us set $t_{0}=t$ and $t_{N+1}=s$. Then:

$$
\begin{aligned}
\Psi\left(s, X_{s}, Y_{s}\right)-\Psi(t, x, y)=\sum_{i=0}^{N} & {\left[\Psi\left(t_{i+1}, X_{t_{i+1}}, Y_{t_{i}}\right)-\Psi\left(t_{i}, X_{t_{i}}, Y_{t_{i}}\right)\right] } \\
& +\sum_{i=1}^{N}\left[\Psi\left(t_{i}, X_{t_{i}}, Y_{t_{i}}\right)-\Psi\left(t_{i}, X_{t_{i}}, Y_{t_{i-1}}\right)\right]
\end{aligned}
$$


Since on $Y$ is constant on $\left[t_{i}, t_{i+1}\right)$ for $i=0,1, \ldots, N$ by the results known for Ito diffusions $\Psi\left(t_{i+1}, X_{t_{i+1}}, Y_{t_{i}}\right)-\Psi\left(t_{i}, X_{t_{i}}, Y_{t_{i}}\right)=\int_{t_{i}}^{t_{i+1}} A_{0}^{u} \Psi\left(r, X_{r}, Y_{r}\right) d r$. Hence:

$$
\mathbb{E}_{t x y}\left\{\sum_{i=0}^{N}\left[\Psi\left(t_{i+1}, X_{t_{i+1}}, Y_{t_{i}}\right)-\Psi\left(t_{i}, X_{t_{i}}, Y_{t_{i}}\right)\right]\right\}=\int_{t}^{s} A_{0}^{u} \Psi\left(r, X_{r}, Y_{r}\right) d r .
$$

To finish the proof we notice, that we can write:

$$
\mathbb{E}_{t_{i}, y}\left[f\left(t_{i}+1\right)\right]=\lambda(y) \mathbb{E}_{t_{i}}\left[\int_{t_{i}}^{t_{i+1}} f(t) d t\right]
$$

for a continuous function $f(t)$. Then

$$
\begin{aligned}
\mathbb{E}_{t_{i-1}, y}\left[\Psi\left(t_{i}, X_{t_{i}}, Y_{t_{i}}\right)-\Psi\left(t_{i}, X_{t_{i}}, y\right)\right] & \\
& =E_{t_{i-1}, y}\left[E_{t_{i}}\left[\Psi\left(t_{i}, X_{t_{i}}, Y_{t_{i}}\right)-\Psi\left(t_{i}, X_{t_{i}}, y\right)\right]\right] \\
& =\mathbb{E}_{t_{i-1}, y}\left[H \Psi\left(t_{i}, X_{t_{i}}, y\right) / \lambda(y)\right] \\
& =\mathbb{E}_{t_{i}}\left[\int_{t_{i-1}}^{t_{i}} H \Psi\left(t, X_{t}, Y_{t}\right)\right]
\end{aligned}
$$

Implicitly we conditioned by the Wiener process $W$ which is however independent of $Y$ therefore we omitted it in the notation. Now using the strong Markov property of $Y$ we get

$$
\left.\mathbb{E}_{t x y}\left\{\sum_{i=1}^{N}\left[\Psi\left(t_{i}, X_{t_{i}}, Y_{t_{i}}\right)-\Psi\left(t_{i}, X_{t_{i}}, Y_{t_{i-1}}\right)\right]\right\}=\mathbb{E}_{t x y}\left[\int_{t}^{s} H \Psi\left(r, X_{r}, Y_{r}\right) d r\right)\right] .
$$

3.4. Optimal Hedging Strategies. In this subsection we solve the HJB equation (28) in the case of the exponential utility function $U(x)=-e^{-\alpha x}$ with $\alpha>0$. We can get a explicit solution in this case by linearizing the HJB equation by a Hopf-Cole transformation. We hypothesize the form of the solution as $V(t, x, y)=-e^{-\alpha x} F(t, y)$. Substituting in (28) we get:

$$
F(t, y)\left(\frac{1}{2} \frac{\mu^{2}(y)}{\sigma^{2}(y)}\right)=\frac{\partial F}{\partial t}(t, y)+G F
$$

with the terminal condition $F(T, y)=1$. The solution of this equation is given by the so-called Feynman-Kac formula. Indeed, its right hand side is the backward evolution operator of $Y$ (the justification will be given below). Such a formula already appeared in [?] where the zero order term found in the exponential was called the traded risk premium. The solution is:

$$
F(t, y)=\mathbb{E}_{t y}\left[e^{-\int_{t}^{T} \frac{1}{2} \frac{\mu^{2}\left(Y_{s}\right)}{\sigma^{2}\left(Y_{s}\right)} d s}\right] .
$$

Substituting out we see that the candidate for the expression of the value function solving the HJB equation (28) with exponential utility is

$$
V(t, x, y)=-e^{-\alpha x} \mathbb{E}_{t y}\left[e^{-\int_{t}^{T} \frac{1}{2} \frac{\mu^{2}\left(Y_{s}\right)}{\sigma^{2}\left(Y_{s}\right)} d s}\right],
$$


the corresponding optimal trading strategy being given by the time varying Sharpe ratio

$$
\phi_{t}=\frac{\mu\left(Y_{t-}\right)}{\alpha \sigma^{2}\left(Y_{t-}\right)}
$$

where $Y_{t-}=\lim _{h \backslash 0} Y_{t-h}$ is the left hand limit assuring the predictability of the trading strategy. To check that the candidate solution is indeed the optimal solution of the optimization problem we need to check if it satisfies the verification theorem ([?]), and hence is a bona-fide optimal solution of the original problem. We will proceed to do this for the case when $Y$ is the jump Markov storm model.

Proposition 3.4. If $\sigma(y)>\epsilon>0$ for all $y$ then the trading strategy $\phi$ is the optimal trading strategy in the utility maximization problem with $Y$ being the jump Markov process storm model.

Proof. We first show that the strategy $\phi$ has all moments and therefore is admissible:

$$
\begin{aligned}
\mathbb{E}\left[\int_{0}^{T}\left|\phi_{t}\right|^{m} d t\right] & =\mathbb{E}\left[\int_{0}^{T}\left|\frac{\mu\left(Y_{t}\right)}{\sigma^{2}\left(Y_{t}\right) \alpha}\right| d t\right] \\
& \leq K \mathbb{E}\left[\int_{0}^{T}\left|Y_{t}\right|^{m} d t\right] \\
& =(K T) \mathbb{E}\left[\frac{1}{T} \int_{0}^{T}\left|Y_{t}\right|^{m} d t\right] \\
& <(K T) \mathbb{E}\left[\left|Y_{\infty}\right|^{m}\right]
\end{aligned}
$$

where the last inequality follows from the ergodicity and existence of all moments of the process $Y$ (see Theorem 2.7). It remains to show that $V \in \mathcal{D}$. We will show that $V$ satisfies the conditions of the proposition 3.3. It is easy to see that all the conditions are satisfied if and only if

$$
\mathbb{E}_{t y}\left[\exp \left(-\frac{1}{2} \int_{t}^{T} \frac{\mu^{2}\left(Y_{s}\right)}{\sigma^{2}\left(Y_{s}\right)} d s\right)\right]<C|y|^{m}
$$

which is obvious since the left hand side is bounded.

We can solve the HJB equation (28) along the same lines in the case of the power utility function $U(x)=x^{\alpha} / \alpha$ with $\alpha<1$. Again we search for a value function in the form $V(t, x, y)=$ $\left(x^{\alpha} / \alpha\right) F(t, y)$, and substituting out we get the linearized equation:

$$
F(t, y)\left(\frac{\alpha}{2(1-\alpha)} \frac{\mu^{2}(y)}{\sigma^{2}(y)}\right)=\frac{\partial F}{\partial t}(t, y)+G V
$$

with terminal condition $F(T, y)=1$. Again, invoking the Feynman-Kac formula we get the solution:

$$
F(t, y)=\mathbb{E}_{t y}\left[e^{\int_{t}^{T} \frac{\alpha}{2(1-\alpha)} \frac{\mu^{2}\left(Y_{s}\right)}{\sigma^{2}\left(Y_{s}\right)} d s}\right]
$$

and after substitution, we get the candidate for the solution of (28) with power utility to be:

$$
V(t, x, y)=\frac{x^{\alpha}}{\alpha} \mathbb{E}_{t y}\left[e^{\int_{t}^{T} \frac{\alpha}{2(1-\alpha)} \frac{\mu^{2}\left(Y_{s}\right)}{\sigma^{2}\left(Y_{s}\right)} d s}\right]
$$

with the corresponding candidate

$$
\phi_{t}=\frac{\mu\left(Y_{t-}\right)}{\sigma^{2}\left(Y_{t-}\right)(1-\alpha)} X_{t}
$$


for trading strategy. Verification can be done along the same lines as with exponential utility.

3.5. Utility Maximization with a Derivative. We are ready to move onto maximizing the expected utility with a derivative written on the underlying process $Y$. In precipitation market practically all derivatives have Asian structure since the instantaneous precipitation intensity has little practical importance. Two main types of precipitation derivatives can be encountered. The payoff can depend upon the amount of rain during a given time period $f_{1}(Y)=\left(\int_{t^{\prime}}^{t^{\prime \prime}} Y_{t} d t-K\right)^{+}$, or upon the amount of time it rains (does not rain) during a given time period $f_{2}(Y)=\left(\int_{t^{\prime}}^{t^{\prime \prime}} 1_{\left\{Y_{t}>\epsilon\right\}} d t-K\right)^{+}$, where $\epsilon$ is the minimal precipitation intensity that constitutes a "rainy day". For simplicity we first assume $K=0$, later we will show how we can extend our results to the case $K>0$. Under this simplifying assumption both payoffs can be expressed under a common framework $\xi=\int_{t^{\prime}}^{t^{\prime \prime}} h\left(Y_{s}\right) d s \quad 0 \leq t^{\prime} \leq$ $t^{\prime \prime} \leq T$. For type (1) derivative $h(y)=y$, for type (2) in turn $h(y)=1_{(\epsilon, \infty)}(y)$. The results below also hold for any function $h \geq 0$ with polynomial growth.

When maximizing the utility two points of view have to be considered: the buyer's and the seller's. The buyer's objective function is: $\mathbb{E}\left[U\left(X_{T}+\xi\right)\right]$. The seller's in turn is: $\mathbb{E}\left[U\left(X_{T}-\xi\right)\right]$. We will solve only the buyer's problem. The sellers problem can be solved along the same lines. We transform the optimal portfolio problem with the derivative present to an equivalent problem of optimal investment with random endowment - for which a rather extensive body of literature exists ([?], [?], $[?])$.

Proposition 3.5. The solution $\left(\phi^{*}, V^{*}\right)$ of buyer's problem is the same as the solution $\left(\hat{\phi}^{*}, \hat{V}^{*}\right)$ of the following random endowment optimal investment problem:

$$
\begin{gathered}
\max _{\phi} \mathbb{E}\left[U\left(\hat{X}_{T}\right)\right] \\
d \hat{X}_{t}=g\left(t, Y_{t}\right) d t+\hat{\phi}_{t}\left(\mu\left(Y_{t}\right) d t+\sigma\left(Y_{t}\right) d W_{t}\right)
\end{gathered}
$$

where $g(t, y)=1_{\left(t^{\prime}, t^{\prime \prime}\right)}(t) h(y)$.

Proof. Let $\phi$ be an admissible strategy for the buyer's problem. Let us denote $V(\phi), X(\phi), \hat{V}(\phi)$, $\hat{X}(\phi)$ the value functions and wealth processes in the original problem and (32) respectively corresponding to the strategy $\phi$. It is clear that $\phi$ is an admissible strategy for problem (32) and $V(\phi) \leq \hat{V}(\phi) \leq \hat{V}^{*}$ and therefore $V^{*} \leq \hat{V}^{*}$. Now let us consider the optimal strategy $\hat{\phi}^{*}$ for the problem (32) and define a new strategy $\psi=\left(\psi^{0}, \psi^{1}\right)$; it is necessary to look at both components of the strategy, the amount of money invested in the risky asset and the riskless bond. Let $\psi^{1}=\left(\hat{\phi}^{*}\right)^{1}$ and $\psi_{t}^{0}=\left(\hat{\phi}^{*}\right)_{t}^{0}-\int_{0}^{t} g\left(s, Y_{s}\right) d s$. Then $\hat{X}(\psi)$ satisfies $d \hat{X}_{t}=\psi_{t}^{1}\left(\mu\left(Y_{t}\right) d t+\sigma\left(Y_{t}\right) d W_{t}\right)$ and therefore $X_{T}(\psi)=\hat{X}_{T}\left(\phi^{*}\right)-\int_{0}^{T} g\left(t, Y_{t}\right) d t$ and hence

$$
V^{*} \geq V(\psi)=\mathbb{E}\left[U\left(X_{T}(\psi)+\int_{0}^{T} g\left(t, Y_{t}\right) d t\right)\right]=\mathbb{E}\left[U\left(X_{T}\left(\phi^{*}\right)\right]=\hat{V}^{*}\right.
$$

Proposition 3.6. The solution $\left(\phi^{*}, V^{*}\right)$ of seller's problem is the same as the solution $\left(\hat{\phi}^{*}, \hat{V}^{*}\right)$ of the following random endowment optimal investment problem: 


$$
\begin{gathered}
\max _{\phi} \mathbb{E}\left[U\left(\hat{X}_{T}\right)\right] \\
d \hat{X}_{t}=-g\left(t, Y_{t}\right) d t+\hat{\phi}_{t}\left(\mu\left(Y_{t}\right) d t+\sigma\left(Y_{t}\right) d W_{t}\right)
\end{gathered}
$$

where $g\left(t, Y_{t}\right)=1_{\left(t^{\prime}, t^{\prime \prime}\right)}(t) h\left(Y_{t}\right)$.

The proof of this proposition goes along the same lines as proposition (3.5).

Now we can write the HJB equation for problem (32):

$$
0=\frac{\partial V}{\partial t}+\frac{\partial V}{\partial x} g(t, y)+G V+\sup _{u}\left\{u \mu(y) \frac{\partial V}{\partial x}+\frac{1}{2} u^{2} \sigma^{2}(y) \frac{\partial^{2} V}{\partial x^{2}}\right\}
$$

We can perform the maximization with respect to $u$ explicitly to get the candidate optimal decision $u^{*}$ and explicit HJB equation:

$$
\begin{gathered}
u^{*}=-\frac{\mu(y)}{\sigma^{2}(y)} \frac{\partial V / \partial x}{\partial^{2} V / \partial x^{2}} \\
0=\frac{\partial V}{\partial t}+\frac{\partial V}{\partial x} g(t, y)+G V-\frac{1}{2} \frac{\mu^{2}(y)}{\sigma^{2}(y)} \frac{(\partial V / \partial x)^{2}}{\partial^{2} V / \partial x^{2}}
\end{gathered}
$$

with the terminal condition $V(T, x, y)=U(x)$. Specifically for the jump Markov process storm model (36) becomes:

$$
0=\frac{\partial V}{\partial t}+\frac{\partial V}{\partial x} g(t, y)+\lambda(y) \int_{R}[V(t, x, z)-V(t, x, y)] \Pi(y, d z)-\frac{1}{2} \sigma^{2}(y) \frac{(\partial V / \partial x)^{2}}{\partial^{2} V / \partial x^{2}}
$$

The domain $\mathcal{D}$ for this equation is the same as for the equation (28).

The exponential utility allows us to separate variables in the equation. As before we search for an expression of the value function in the form $V(t, x, y)=-e^{-\alpha x} F(t, y)$. The HJB equation then reduces to:

$$
F(t, y)\left(\alpha g(t, y)+\frac{1}{2} \frac{\mu^{2}(y)}{\sigma^{2}(y)}\right)=\frac{\partial F}{\partial t}(t, y)+G F
$$

with terminal condition $F(T, y)=1$, and using the Feynman-Kac representation formula we obtain:

$$
F(t, y)=\mathbb{E}_{y}\left[e^{-\int_{t}^{T}\left(\alpha g\left(s, Y_{s}\right)+\frac{1}{2} \frac{\mu^{2}\left(Y_{s}\right)}{\sigma^{2}\left(Y_{s}\right)}\right) d s}\right] .
$$

Thus the candidate solution is:

$$
V(t, x, y)=-e^{-\alpha x} \mathbb{E}_{y}\left[e^{-\int_{t}^{T}\left(\alpha g\left(s, Y_{s}\right)+\frac{1}{2} \frac{\mu^{2}\left(Y_{s}\right)}{\sigma^{2}\left(Y_{s}\right)}\right) d s}\right]
$$

with the corresponding trading strategy $\phi_{t}=\mu(y) /\left(\sigma^{2}(y) \alpha\right)$. We have a verification result: 
Proposition 3.7. If $\sigma(y)>\epsilon>0$ for all $y$ the trading strategy $\phi$ is the optimal trading strategy in the utility maximization problem 32 with $Y$ being the jump Markov process storm model.

The proof of this verification result is analogous to the proof of Proposition 3.4.

The situation is more complicated in the case of power utility. It does not possess the convenient separability property of the exponential utility therefore adding the derivatives causes the separation of variables argument to fail. A lot of attention has been given to this problem. For example, existence of the solution has been proved in a very general setting in ([?]). Henderson ([?]) derived lower and upper bounds for the value function in the case where the derivative payoff is small compared to the wealth of the agent. Her approach is based on convex duality (to obtain the upper bound) and expansion of the utility around the no-derivative solution (to get the rate of convergence of the lower and an upper bound). We will apply the approach of Henderson, to obtain bounds on the value function in our case.

$\diamond$ Lower Bound A trivial lower bound is given by the value function of the problem without the derivative since the derivative payoff is always non-negative. In the present situation, we can improve this bound by using Proposition 3.5. We use the optimal strategy from the problem without the derivative (24) in the transformed random endowment problem (32). An elementary calculation shows that the value function for this strategy is:

$$
\begin{aligned}
& V_{L}(0, x, y)=\frac{1}{\alpha} E_{y}\left\{\exp \int_{0}^{T} \frac{\mu^{2}\left(Y_{t}\right)}{\sigma^{2}\left(Y_{t}\right)} \frac{1}{1-\alpha}\left(1-\frac{1}{2(1-\alpha)}\right) d t\right. \\
& \left.\quad\left[x+\int_{0}^{T} g\left(t, Y_{t}\right) \exp \left(-\int_{0}^{t} \frac{\mu^{2}\left(Y_{s}\right)}{\sigma^{2}\left(Y_{s}\right)} \frac{1}{1-\alpha}\left(1-\frac{1}{2(1-\alpha)}\right) d s-\frac{1}{1-\alpha} \int_{0}^{t} \frac{\mu\left(Y_{s}\right)}{\sigma\left(Y_{s}\right)} d W_{s}\right)\right]\right\}^{\alpha}
\end{aligned}
$$

which by Proposition 3.5 is greater than the no-derivative value function.

$\diamond$ Upper bound We get an upper bound by employing the convex duality theory. Started by Karatzas et. al ([?]) in the Ito processes setting, it was extended by Cvitanic ([?]) to the more general semimartingale setting. Our upper bound is a direct consequence of the main result of [?] when we choose a particular equivalent martingale measure $\mathbb{Q}^{0}$. A natural candidate is the one suggested by Girsanov's theorem. It is defined by its density with respect to $\mathbb{P}$ :

$$
\frac{d \mathbb{Q}^{0}}{d \mathbb{P}}=\exp \left(-\int_{0}^{T} \frac{\mu\left(Y_{s}\right)}{\sigma(Y s)} d W_{s}-\frac{1}{2} \int_{0}^{T} \frac{\mu^{2}\left(Y_{s}\right)}{\sigma^{2}\left(Y_{s}\right)} d s\right)
$$

Clearly the measure $\mathbb{Q}^{0}$ makes the traded asset price $S$ and therefore the wealth process $X$, a martingale; Moreover, it is equivalent to $\mathbb{P}$. Hence, it is in $\Delta$. We can get the value of the dual objective function for this measure:

$$
\mathbb{E}\left[\tilde{U}\left(y \frac{d \mathbb{Q}}{d \mathbb{P}}\right)\right]=\frac{1-\alpha}{\alpha} y^{\frac{\alpha}{\alpha-1}} A \quad \text { where } \quad A=\mathbb{E}\left[\exp \left(\frac{1}{2} \frac{\alpha}{(1-\alpha)^{2}} \int_{0}^{T} \frac{\mu^{2}\left(Y_{s}\right)}{\sigma^{2}\left(Y_{s}\right)} d s\right)\right] .
$$

Therefore: $D(y)=\frac{1-\alpha}{\alpha} y^{\frac{\alpha}{\alpha-1}} A+y\left(x+\mathbb{E}^{0}[\xi]\right)$, and the upper bound is obtained by minimizing $D(y)$ over $y$. We get:

$$
\frac{1-\alpha}{\alpha}\left(x+\mathbb{E}^{0}[\xi]\right)^{\alpha} A^{1-\alpha}=V_{0}(t, x, y)\left(1+\frac{\mathbb{E}^{0}[\xi]}{x}\right)^{1-\alpha}
$$


where $V_{0}$ is the value function of the problem (24) with no derivative present. It is easy to see that these bounds converge as the wealth level becomes large compared to the derivative payoff.

3.6. The Indifference Prices. As we mentioned in the introduction the solution to the optimization problems without a derivative claim and with it allow us to set a price on the derivative claim by the principle of indifference. Let us denote $V_{0}$ the value function of (24), $V_{\xi}$ the value function of (32) and $p$ the price of the derivative claim with payoff $\xi$. The indifference principle states $V_{0}(0, x+p, y)=$ $V_{\xi}(0, x, y)$. Solving this implicit equation for $p$ will allow us to price the derivative. We will be able to give quite an explicit formula in the exponential utility case. With the power utility we will only be able to give bounds on the price, as expected.

Using the results of Subsection 3.4 and plugging the value functions in the indifference equation we obtain for the exponential utility:

$$
p=\frac{1}{\alpha} \ln \frac{\mathbb{E}_{y}\left[e^{\left.-\int_{0}^{T} \frac{1}{2} \frac{\mu^{2}\left(Y_{s}\right)}{\sigma^{2}\left(Y_{s}\right)}\right] s}\right]}{\mathbb{E}_{y}\left[e^{-\int_{0}^{T}\left(\alpha g\left(s, Y_{s}\right)+\frac{1}{2} \frac{\mu^{2}\left(Y_{s}\right)}{\sigma^{2}\left(Y_{s}\right)}\right) d s}\right]} .
$$

Notice that this value of $p$ is independent of the initial wealth. This is very specific to the particular choice of the exponential utility function. Also $p$ is in practically closed form since it can be easily evaluated by Monte Carlo simulation of the precipitation process $Y$, whose sample paths can be simulated without error.

In the case of power utility, we get bounds based on the bounds on the value function:

$$
\left(\frac{\alpha V_{L}(0, x, y)}{\mathbb{E}_{t y}\left[e^{\int_{t}^{T} \frac{\alpha}{2(1-\alpha)} \frac{\mu^{2}\left(Y_{s}\right)}{\sigma^{2}\left(Y_{s}\right)} d s}\right]}\right)^{1 / \alpha}-x \leq p \leq x\left[1-\left(1+\frac{\mathbb{E}_{y}\left[\int_{0}^{T} g\left(t, Y_{t}\right) d t \frac{d Q^{0}}{d P}\right]}{x}\right)^{\frac{\alpha-1}{\alpha}}\right] .
$$

3.7. Derivatives with Non-zero Strikes. To simplify the notation, in the previous sections we only considered derivatives with the payoff $\xi$ of the form: $\xi=\int_{t^{\prime}}^{t^{\prime \prime}} h\left(Y_{s}\right) d s \quad 0 \leq t^{\prime} \leq t^{\prime \prime} \leq T$. We will show that our results directly extend to the payoffs of the form $\xi=\left(\int_{t^{\prime}}^{t^{\prime \prime}} h\left(Y_{s}\right) d s-K\right)^{+} \quad 0 \leq t^{\prime} \leq$ $t^{\prime \prime} \leq T$ where the derivative starts paying off only after a certain strike amount $K>0$ is reached. To deal with this case let us define a process $R_{t}=\int_{t^{\prime}}^{t} h\left(Y_{s}\right) d s$ for $t \geq t^{\prime}$ and 0 otherwise and a payoff rate function $\hat{h}\left(Y_{t}, R_{t}\right)=1_{\left\{R_{t}>K\right\}} h\left(Y_{t}\right)$. It is easy to see that the payoff $\xi$ can be written in terms of $\hat{h}$ as $\xi=\int_{t^{\prime}}^{t^{\prime \prime}} \hat{h}\left(Y_{s}, R_{s}\right) d s \quad 0 \leq t^{\prime} \leq t^{\prime \prime} \leq T$

We were able to eliminate the strike $K$ at the expense of increasing the dimension of the underlying by one. Let us denote by $\hat{Y}=(Y, R)$ this new underlying process. The optimization problem we are facing now is as follows:

$$
\max _{\phi} \mathbb{E}\left[U\left(X_{T}\right)\right]
$$

subject to: $d X_{t}=\hat{g}\left(t, \hat{Y}_{t}\right) d t+\phi_{t}\left(\mu\left(Y_{t}\right) d t+\sigma\left(Y_{t}\right) d W_{t}\right)$ where $\left.\hat{g}(t, y, r)=1_{\left(t^{\prime}, t^{\prime \prime}\right)}(t) \hat{h}(y, r)\right)$. Clearly $\hat{Y}$ is a random evolution with infinitesimal generator $\hat{G} f(y, r)=G+\frac{\partial f}{\partial r} g(t, y)$. Because $\hat{Y}$ 
is a random evolution and hence a Markov process, we can apply the theory developed in previous sections to the solution of problem (40) and obtain equivalent results.

For the exponential utility the value function is:

$$
V(t, x, y, r)=-e^{-\alpha x} \mathbb{E}_{y, r}\left[e^{-\int_{t}^{T}\left(\alpha \hat{g}\left(s, \hat{Y}_{s}\right)+\frac{1}{2} \frac{\mu^{2}\left(Y_{s}\right)}{\sigma^{2}\left(Y_{s}\right)}\right) d s}\right]
$$

with the corresponding trading strategy: $\phi_{t}=\frac{\mu(y)}{\sigma^{2}(y) \alpha}$ and the indifference price:

$$
p=\frac{1}{\alpha} \ln \frac{\mathbb{E}_{y}\left[e^{-\int_{0}^{T} \frac{1}{2} \frac{\mu^{2}\left(Y_{s}\right)}{\sigma^{2}\left(Y_{s}\right)} d s}\right]}{\mathbb{E}_{y, r}\left[e^{-\int_{0}^{T}\left(\alpha \hat{g}\left(s, \hat{Y}_{s}\right)+\frac{1}{2} \frac{\mu^{2}\left(Y_{s}\right)}{\sigma^{2}\left(Y_{s}\right)}\right) d s}\right]}
$$

For the power utility we again get bounds based on the bounds on the value function:

$$
\left(\frac{\alpha V_{L}(0, x, y)}{E_{t y}\left[e^{\int_{t}^{T} \frac{\alpha}{2(1-\alpha)} \frac{\mu^{2}\left(Y_{s}\right)}{\sigma^{2}\left(Y_{s}\right)} d s}\right]}\right)^{1 / \alpha}-x \leq p \leq x\left[1-\left(1+\frac{E_{y}\left[\int_{0}^{T} \hat{g}\left(t, \hat{Y}_{t}\right) d t \frac{d Q^{0}}{d P}\right]}{x}\right)^{\frac{\alpha-1}{\alpha}}\right] .
$$

\section{A Practical application}

In this section we apply the techniques developed in this paper to price rainfall sample contracts which we chose for the purposes of this study. We chose a location in Norway and electric power prices because Norway produces most of its electricity from hydroelectric powerplants (hence power can serve as traded asset in our model) and because of the success of deregulation and the availability of wholesale electricity prices in the NordPool.

Consider a sample contract: a call option on the amount of rainfall during June and July 2004, as recorder by Bergen weather station in Norway with a strike of $\mathrm{K} \mathrm{mm}$ and tick price $1 \mathrm{NOK}$, i.e. the seller of the contract pays to the buyer one NOK for each millimeter of cumulative rainfall above $\mathrm{K}$ $\mathrm{mm}$ during June and July 2004 in Bergen, Norway.

We need to fit the jump Markov model to rainfall in Bergen, find a traded asset influenced by the amount of rainfall in Bergen in June and July, fit a model of the type considered in the paper, determine the exact nature of the functions $\mu$ and $\sigma$ in (20), and calibrate the model to empirical data. The Norwegian Meteorological Institute kindly provided us with high-frequency rainfall intensity data records from the "pluviometer" equipped weather station in Bergen for calendar year 2002. Pluviometers record times (with one minute precision) of tips of a bucket with known (small) volume, and therefore provides practically continuous time observations of the rainfall intensity process. A time series plot of these data is given in Figure 3. We examined the influence of the amount of rainfall on prices of several instruments liquidly traded on the NordPool power exchange, looking for a strong relationship between Fourth Quarter 2002 forward power contract and rainfall in Bergen during the course of the whole year. NordPool is a name of a common electric power exchange of four Nordic countries: Norway, Sweden, Finland and Denmark, established after the liberalization of their power markets. Nowadays it is one of the most liquid European power exchanges offering spot and forward 


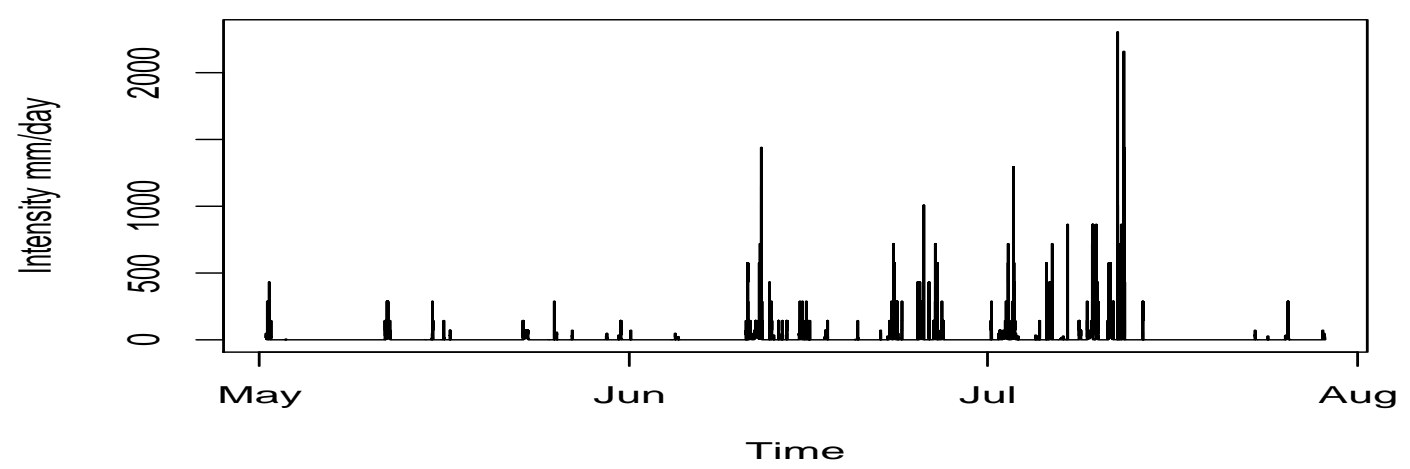

FIGURE 3. Bergen, Norway May-July 2002 rainfall intensity

electricity products. Finally we calibrated the functional form of $\mu$ and $\sigma$ to the data, and we used Monte Carlo computations to arrive at utility indifference prices for the contracts. The details of the exercise follow.

4.1. Bergen Rainfall Model. Rainfall data from May to July 2002 were selected to fit our rainfall model. The period was chosen to closely follow the underlying period of the weather derivative since the rainfall characteristics vary over the course of the year ([?]). The data are plotted in Figure 3. The maximum likelihood parameters estimates for the multiple storm model fitted to these data are listed in Table 1. We computed approximate confidence intervals using result 6 . of the theorem 2.2. We could not obtain confidence intervals for parameters of the process $\bar{\lambda}_{1}$ only for the mean of its limiting distribution. The maximum likelihood mean estimate is 11.36 with approximate $95 \%$ c.i. $(10.68,12.05)$. The confidence intervals are quite tight given the short period of data used for fitting the model. This is because of the very high time resolution of the data (the sample length was 2150). Due to the measurement methodology of pluviometers, the rainfall intensity is always greater than 0 . The cutoff level under which the rainfall intensity is considered to be 0 was set to $1 \mathrm{~mm} /$ day.

TABLE 1. Parameter estimates for Bergen data

\begin{tabular}{|l|ll|l|}
\hline Parameter & Value & C.I. $(95 \%)$ \\
\hline$\lambda_{1}$ & 78.6 & day $^{-1}$ & see text \\
$q_{d}$ & 0.85 & day $^{-1}$ & see text \\
$q_{p}$ & 5.03 & day $^{-1}$ & see text \\
$\lambda_{2}^{(I)}$ & 0.00 & day $^{-1}$ & $(-0.081,0.081)$ \\
$\lambda_{2}^{(I I)}$ & 2.82 & $\mathrm{~mm}^{-1}$ & $(2.702,2.935)$ \\
$\lambda_{u}$ & 0.012 & daymm $^{-1}$ & $(0.0116,0.0131)$ \\
$\lambda_{d}$ & 0.011 & daymm $^{-1}$ & $(0.0102,0.0118)$ \\
\hline
\end{tabular}


4.2. The Influence of Bergen's Rainfall on Nordpool Power Prices. We examined several products traded on NordPool power exchange to see whether their prices were influenced by rainfall in Bergen. Of the examined products forward Fourth Quarter Baseload 2002 (Q402) seemed to be the electricity product most influenced by rainfall during the summer period. Fourth Quarter 2002 Baseload forward contract is a contract for delivery of constant power capacity on Norwegian power grid during 24 hours, every day of the period starting 1/10/2002 ending 31/12/2002. The price of this contract is reported with daily granularity. The plot of precipitation intensity together with daily prices of Q402 is given in Figure 4. It is clear from these plots taht there is an apparent upwards trend in the prices during dry periods and downward trend during wet periods. A commonly used model for
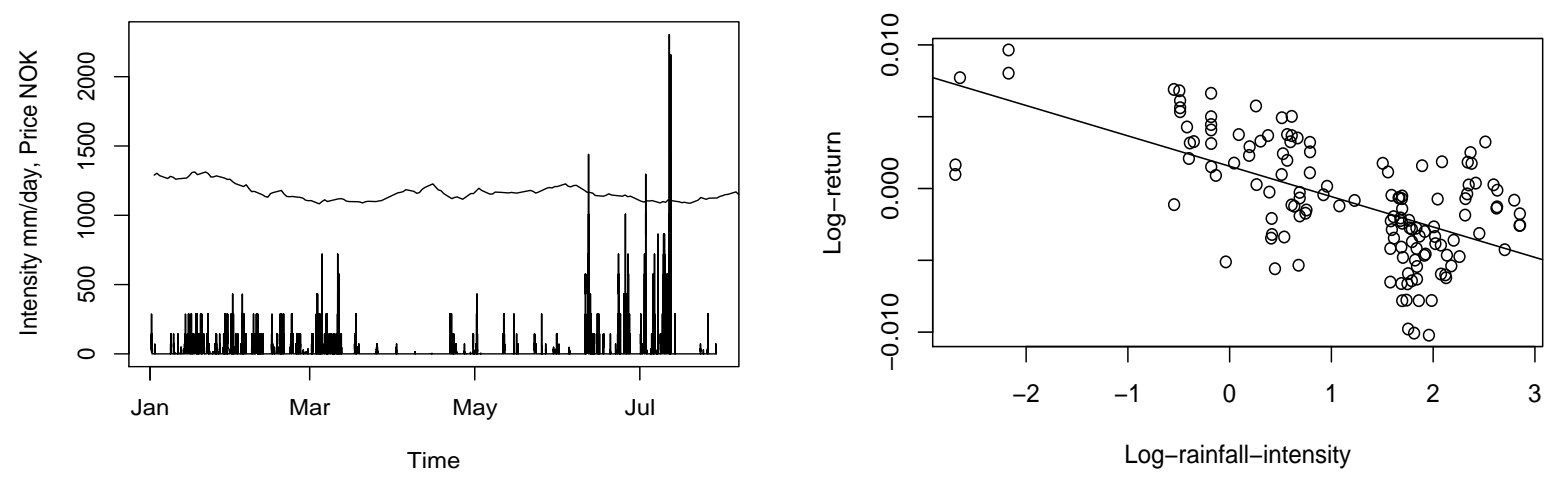

FIGURE 4. Q402 NordPool forward price and Bergen, Norway January-July 2002 rainfall intensity (left) and Bergen log-rainfall-intensity vs. Q402 log-returns.

electricity forward prices in the energy derivatives literature (see for example [?]) is the geometric Brownian motion described by the stochastic differential equation (20). To complete the model we need to express the relationship between power price and rainfall via the functional forms of the drift and volatility coefficients $\mu$ and $\sigma$ of the stochastic differential equation (20) driving the dynamics of the Q402 forward contract. Let us denote by $F_{d}$ the price of Q402 contract on a day $d$, and let us denote by $R_{d}$ the aggregate amount of rainfall in Bergen on that day. We estimate the drift coefficient of $\bar{\mu}_{d}$ on day $d$ by the average of Q402 log-returns on over an 11 day window centered on day $d$. Accordingly, we estimate the rainfall intensity $\bar{R}_{d}$ on the same day by the average rainfall over the same period. Special treatment is necessary for weekends. They are considered as one day and are included in the rainfall intensity average and not in price log-returns average as there are no prices reported on weekends. The relationship between $\bar{R}_{d}$ and $\bar{\mu}_{d}$ is non-linear. Taking logarithm of $\bar{R}_{d}$ makes the relationship linear as we can see from Figure 4. From this analysis, we obtain a functional dependence of Q402 drift coefficient on the rainfall intensity: $\mu(y)=a * \ln (\epsilon+y)+b$ where $\epsilon$ represents the cutoff level for no rainfall $a, b$ are obtained by ordinary least squares regression. The values of the three parameters together with the estimate of the volatility are given in Table 2. (Where 
relevant, the confidence intervals are given.) Rather wide confidence intervals are a direct consequence of scarcity of data. The power markets are rather young and substantial historical price track record has not been generated yet.

TABLE 2. Parameter estimates for Q402 drift and volatility

\begin{tabular}{|l|l|l|}
\hline Parameter & Value & C.I. $(95 \%)$ \\
\hline$a$ & -0.55 & $(-0.67,-0.43)$ \\
$b$ & 0.40 & $(0.20,0.60)$ \\
$\epsilon$ & 0.10 & N/A \\
$\sigma$ & 0.20 & $(0.18,0.22)$ \\
\hline
\end{tabular}

4.3. Utility Indifference Price for Bergen Rainfall Call. We use the price formula derived in Subsection 3.7 and we rely on Monte Carlo simulation of the rainfall process to compute the expectations and put a price on the sample contract. Since we derived exact pricing formulae in this case, we assume that buyer and seller have exponential utility functions. Also, our bound on the seller's price would be indentically infinity which is another reason to work with exponential utility. Table 5 lists the buyer/seller prices for various strike prices and risk aversion levels. It compares them to the utility indifference prices without trading the power contract, and shows the relative decrease in the price due to hedging with the electricity contract. Also, we give a risk-neutral price under a measure which makes price process $S$ a martingale and preserves the historical statistics of the rainfall process $Y$. As expected the buyer's price without hedging is lower than the risk-neutral price while the seller's is higher. The difference increases with increasing risk aversion (i.e. concavity of utility function). Both sellers's and buyer's price with power hedging is lower than without it. The gap between buyer's and seller's price decreases but remains positive. It is interesting to notice, however that for low risk aversion levels, the seller price with hedging is lower than the buyer price without hedging. Hence, if in reality a seller with knowledge and access to power markets meets a buyer without the access or knowledge a mutually acceptable weather-derivative deal can be done if the seller hedges herself in the power market.

A word of warning is appropriate at this point. Applying the method in practice must be done with caution. The rainfall model parameters but mainly the rainfall influence on traded asset price evolution cannot be estimated without error. As could be seen from the approximate confidence intervals computation the uncertainty can be as much as $20 \%$. The resulting uncertainty in price can be comparable to the bid/ask spread.

\section{REFERENCES}

[1] C.W. Anderson, N. Mole, and S. Nadarajah. A switching poisson process model for high concentrations in short-range atmospheric dispersion. Atmospheric Environment, 31(6):813-824, 1997.

[2] P. Billingsley. Statistical Inference for Markov Processes. The University of Chicago Press, 1961.

[3] R. Carmona and A. Danilova. Optimal utility hedging for markets with basis risk: The case of temperature options. submitted for publication, 2003.

[4] R.E. Chandler. A spectral method for estimationg parameters in rainfall models. Bernoulli, 3(3):301-322, 1997.

[5] L. Clewlow and Ch. Strickland. Valuing energy options in a one factor model fitted to forward prices. working paper, 1999. 
FIGURE 5. Bergen rainfall pricing results

\begin{tabular}{|c|c|c|c|c|c|c|c|}
\hline \multirow[b]{3}{*}{ Strike } & \multirow{3}{*}{$\begin{array}{c}\text { Risk } \\
\text { aversion }\end{array}$} & \multicolumn{4}{|c|}{ Utility indifference price } & \multirow{3}{*}{$\begin{array}{l}\text { Seller's } \\
\text { hedging } \\
\text { discount }\end{array}$} & \multirow{3}{*}{$\begin{array}{c}\text { Risk } \\
\text { neutral } \\
\text { price }\end{array}$} \\
\hline & & \multicolumn{2}{|c|}{ With power hedge } & \multicolumn{2}{|c|}{ Without power hedge } & & \\
\hline & & Seller & Buyer & Seller & Buyer & & \\
\hline \multirow[t]{3}{*}{200} & 0.1 & 232.68 & 33.54 & 235.20 & 36.51 & $99 \%$ & 145.97 \\
\hline & 0.01 & 158.63 & 117.26 & 167.00 & 124.85 & $95 \%$ & 145.21 \\
\hline & 0.001 & 133.62 & 129.96 & 140.99 & 137.15 & $95 \%$ & 139.05 \\
\hline \multirow[t]{3}{*}{250} & 0.1 & 167.90 & 27.94 & 169.87 & 29.95 & $99 \%$ & 98.98 \\
\hline & 0.01 & 111.48 & 68.15 & 122.32 & 75.69 & $91 \%$ & 96.07 \\
\hline & 0.001 & 84.16 & 80.90 & 91.19 & 87.82 & $92 \%$ & 89.49 \\
\hline \multirow[t]{3}{*}{300} & 0.1 & 174.13 & 11.31 & 177.71 & 12.72 & $98 \%$ & 57.29 \\
\hline & 0.01 & 60.81 & 34.12 & 70.52 & 39.01 & $86 \%$ & 50.58 \\
\hline & 0.001 & 43.82 & 41.43 & 49.52 & 46.84 & $88 \%$ & 48.16 \\
\hline \multirow[t]{3}{*}{350} & 0.1 & 162.90 & 4.37 & 167.38 & 5.18 & $97 \%$ & 26.21 \\
\hline & 0.01 & 24.82 & 14.68 & 28.79 & 17.15 & $86 \%$ & 21.92 \\
\hline & 0.001 & 27.82 & 25.90 & 34.17 & 31.86 & $81 \%$ & 32.99 \\
\hline \multirow[t]{3}{*}{400} & 0.1 & 116.06 & 1.89 & 120.36 & 2.31 & $96 \%$ & 9.09 \\
\hline & 0.01 & 6.37 & 3.60 & 8.20 & 4.61 & $78 \%$ & 5.99 \\
\hline & 0.001 & 6.44 & 6.15 & 8.04 & 7.68 & $80 \%$ & 7.86 \\
\hline \multirow[t]{3}{*}{450} & 0.1 & 14.53 & 0.36 & 17.01 & 0.48 & $85 \%$ & 1.65 \\
\hline & 0.01 & 2.90 & 1.25 & 4.30 & 1.81 & $68 \%$ & 2.65 \\
\hline & 0.001 & 3.27 & 2.97 & 4.98 & 4.50 & $66 \%$ & 4.73 \\
\hline \multirow[t]{3}{*}{500} & 0.1 & 0.38 & 0.07 & 0.54 & 0.10 & $71 \%$ & 0.20 \\
\hline & 0.01 & 0.38 & 0.30 & 0.55 & 0.43 & $70 \%$ & 0.48 \\
\hline & 0.001 & 0.21 & 0.21 & 0.30 & 0.29 & $72 \%$ & 0.30 \\
\hline
\end{tabular}

[6] P.S.P. Cowpertwait. A generalized point process model for rainfall. Proceedings: Mathematical and Physical Sciences, 447(1929):23-37, October 1994.

[7] J. Cvitanic, W. Schachermayer, and H. Wang. Utility maximization in incomplete markets with random endowment. Finance And Stochastics, 5(2):259-272, May 2001.

[8] M.H.A. Davis. Optimal hedging with basis risk. Preprint, page 0, 2000.

[9] D. Duffie, W.H. Fleming, H.M. Soner, and T. Zariphopoulou. Hedging in incomplete markets with HARA utility. Journal of Economic Dynamics \& Control, 21(4-5):753-782, 1997.

[10] R. Carmona (ed). Indifference Picing. Princeton University Press, 2004.

[11] W.H. Fleming and H.M. Soner. Controlled Markov Processes and Viscosity Solutions. Applications of Mathematics 25. Springer-Verlag, 1993.

[12] V.K. Gupta and E.C. Waymire. Multiscaling properties of spatial rainfall and river flow distributions. Journal of Geophysical Research, 95(D3):1999-2010, 1990.

[13] V.K. Gupta and E.C. Waymire. A statistical analysis of mesoscale rainfall as a random cascade. Journal of Applied Meteorology, 32:251-267, 1994. 
[14] V. Henderson. Valuation of claims on nontraded assets using utility maximization. Mathematical Finance, 12(4):351373, October 2002.

[15] V. Henderson and D.G. Hobson. Real options with constant relative risk aversion. Journal of Economic Dynamics \& Control, 27:329-355, 2002.

[16] I. Karatzas, J.P. Lehoczky, S.E. Shreve, and G. Xu. Martingale and duality methods for utility maximization in an incomplete market. SIAM Journal of Control and Optimization, 29(3):702-730, 1991.

[17] N. El Karoui and M. Jeanblanc-Picque. Optimization of consumption with labor income. Finance And Stochastics, 2(4):409-440, 1998.

[18] L. LeCam. A stochastic description of precipitation. Proceedings of the Fourth Berkeley Symposium on Mathematical Statistics and Probability, 3:165-186, 1961. ed. J. Neyman.

[19] J. Mason. Numerical weather prediction. Proceeding of Royal Society in London. Series A., 407:51-60, 1986.

[20] S.P. Meyn and R.L. Tweedie. Markov Chains and Stochastic Stability. Springer-Verlag, 1993.

[21] M. Musiela and T. Zariphopoulou. Indifference prices and related measures. Technical report, 2001.

[22] M. Musiela and T. Zariphopoulou. Pricing and risk management of derivatives written on non-traded assets. preprint, pages $1-39,2001$.

[23] M. Musiela and T. Zariphopoulou. An example of indifference prices under exponential preferences. Technical report, 2003.

[24] C. Onof, R.E. Chandler, A. Kakou, P. Northrop, H.S. Wheater, and V. Isham. Rainfall modelling using poisson-cluster processes: A review of developments. Stochastic Environmental Research and Risk Management, 14:384-411, 2000.

[25] I. Rodriguez-Iturbe, D.R. Cox, and V. Isham. Some models for rainfall based on stochastic point processes. Proceeding of the Royal Society of London. Series A., 410(1839):269-288, April 1987.

[26] I. Rodriguez-Iturbe, D.R. Cox, and V. Isham. A point process model for rainfall: Further developments. Proc. R. Soc. London A, 417:283-298, 1988.

[27] D.S. Wilks and R.L. Wilby. The weather generation game: A review of stochastic weather models. Progress in Physical Geography, 23(3):329-357, 1999.

[28] T. Zariphopoulou. A solution approach to valuation with unhedgeable risks. Finance and Stochastics, 5:61-82, 2002.

Department of Operations Research and Financial Engingeering, Princeton University, Princeton, NJ 08544, Also with the Bendheim Center for Finance and the Applied and Computational Mathematics Program.

E-mail address: rcarmona@princeton.edu

Electrabel S.A., Trading \& Portfolio Management, Boulevard du Rgent 8, Bruxelles 1000, BelGIUM

E-mail address: Pavel.Diko@electrabel.com 